\title{
Imagery and Explanation in the Dynamics of Recall of Intuitive and Scientific Knowledge: Insights from Research on Children's Cosmologies
}

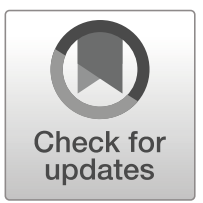

\author{
Tom G. K. Bryce ${ }^{1}$ (D) Eric J. Blown ${ }^{1}$
}

Published online: 30 January 2020

(C) The Author(s) 2020

\begin{abstract}
This article closely examines (a) the representational connotation which is often implicit in many analyses of the scientific knowledge which children have (or have not) acquired when they are asked to say or show what they know and (b) the still common-place presumption that recollections are akin to the extraction of ideas from a mental database. We demonstrate how recent findings in neuroscience reject traditional thinking about the nature of 'representation' and the character of associated imagery and verbal explanation. Researchers have to contend with the fact that concepts must be regarded as flexible, and that memory is dynamic. Such considerations emphasise the creative, rather than the reproductive, nature of remembering, thus calling into question the status of what is thought to be 'grasped' and 'imaged' by those being interviewed, possibly casting some doubt on the status of children's conceptions (and misconceptions) and the categories into which these are sometimes placed in schematic depictions of their understanding. Examples from research on children's cosmologies are used to illustrate the discussion. It is argued that science education researchers endeavouring to uncover what children know, intuitively and scientifically, through interviewing them, face a reconsideration of the theoretical underpinnings to much of their work.
\end{abstract}

Keywords Imagery Clinical interviews · Conceptual representation and change $\cdot$ Mental models Neuroscience $\cdot$ Children's cosmologies

Tom G. K. Bryce

t.g.k.bryce@strath.ac.uk

1 School of Education, University of Strathclyde, Lord Hope Building (level 5), 141 St James Road, Glasgow G4 0LT, UK 


\section{Introduction}

"... every act of perception is to some degree an act of creation and every act of memory is to some degree an act of the imagination.” Edelman and Changeux (2001, p. 56)

We spend much of our lives endeavouring to explain what we mean, clarifying our understanding of things we have encountered, trying to account for the sense they make and the significance they have for us personally. As we explain to others - to teachers and fellow students during instruction, to friends in conversation or to researchers while they interview us-we also explain to ourselves through what Vygotsky described as inner speech' (see Vygotsky 1986). And, as we do so, the mental processes invoke images as well as the words we are trying to use to make things clear to the listener, whether self or other. What we see in our 'mind's eye' usually, but not always, complements our verbal expressions and gives coherence (at least a coherence that works for us) to what we are trying to describe or the ideas we seek to explain. The relationship(s) between the images we 'see' and the explanations we offer as we think and respond to questioning are the focus of this article. Given recent revelations in neuroscience, they require to be understood in terms of what researchers in that field refer to as the flexibility of concepts, the dynamics of memory and its possible non-representational nature. In Bryce and Blown (2016), we gave a first account of how ideas are dynamic and multi-modal, actively created at the point of recall, using children's cosmologies as the focus of our research. The present paper extends that argument, exploiting consciousness as 'a kind of remembered present', in the sense used by Edelman to indicate the dynamic interaction between memory and ongoing perception'. We consider here what is meant by 'non-representational memory' (on which the literature is sparse and awaits further developments in brain research permitting closer analysis of both conscious and unconscious processes) and findings in cognition research which are in support of the creation as well as the inhibition of concepts. The emphasis of our own empirical study, described in this paper, focuses on multi-modal cognitive evidence for the dynamic memory and imagery associated with the coexistence of everyday and scientific concepts.

For the most part, researchers in the field of science education-a quite different domain to neuroscience - especially those concerned with what interviews reveal about children's knowledge and its acquisition have, on the whole, followed the more conventional line of argument that science concepts, once acquired by a young person at any particular stage of schooling, are mental entities which, possessing some functional stability at that time, will be drawn from memory in order to answer questions and solve the problem. The evidence we adduced in Blown and Bryce (2010), and which we will refer to later, supported such functional stability. The researcher's task has been to understand the nature of such concepts - to reveal how the interviewee's ideas are represented as mental entities - and a 'flexibility' of these representations has rarely been a consideration. Indeed, very many reports say nothing about the exact 'constitutional' status of the ideas they detect in pupils' thinking (however scientifically correct or not they are), and they simply record: 'This is what 10 year olds typically say...' (cf. Blown

\footnotetext{
1 "Looked at from the inside, consciousness seems continuously to change, yet at each moment it is all of a piece - what I have called 'the remembered present' - reflecting the fact that all my past experience is engaged in forming my integrated awareness of this single moment" (Edelman 2005, p. 8).
} 
and Bryce 2010). In many investigations, particularly those concerned with basic science concepts, including children's comprehension of ideas in astronomy, it is assumed that when children are remembering, the process is typically thought of as a 'reproductive' act: interviewees are extracting information from their memories and ideas come out, largely 'intact' (This is how I see it/My teacher taught me that.../Mum always used to say that when...). Not infrequently, applied research has been focused on how concepts may be refined or corrected with exposure to appropriate pedagogical initiatives. That said, researchers readily concede that the exact words a child will use to explain their thinking as they say what they remember, and the drawings and models they may produce to amplify their points, will vary from time to time and situation to situation; what is being recalled is not rigidly definable though a semi-structured interview can get at what the pupil knows, especially at the hands of an interviewer who uses Socratic dialogue with sensitivity. In comparing traditional with contemporary, research-informed views of what is actually remembered, this paper looks at what recent findings, particularly those from brain research, mean for investigators concerned to uncover children's knowledge. While the results of neuroscientific investigations and the findings from cognition research do not directly equate - stemming from different levels of analysis - they ought to be compatible with each other, or at least congruent in some ways. Our interview study-reported later-looked for indications of the flexibility to be associated with conceptualisations: we sought to shed light on the intrigues concerning representation and non-representation in how and what people remember. First, we trace the evolution which has taken place in the relevant fields, drawing from published literature in cognition, neuroscience and science education.

\section{Images, Mental Representation and the Dynamics of Memory}

Historically, the links between images and verbal accounts have received somewhat sporadic attention in cognitive psychology and educational research, especially in developmental studies concerned with learning and the accumulation of knowledge. In the aftermath of behaviourism, Paivio $(1971,1986)$ is widely acknowledged as having rejuvenated research into the distinction between verbal and visual representation in our cognitions. His theory of dual-coding recognised the importance of imagery during mental processing; how there can be memory benefits as a result, thus contributing to what is deemed to be what we 'know'. Thomas (2014) has recently commented that dual-coding had "inspired an enormous amount of controversy and experimental research in psychology" and that it was "one of the most influential theories of cognition in the $\left[20^{\text {th }}\right]$ century" (p. 1). Much of the empirical work conducted over these years was indeed of an experimental, or quasi-experimental, nature; relatively little of it was devoted to interviews designed to uncover subjects' knowledge. However, whether it focused on images or verbalised thoughts, cognitive researchers in the later decades of the century challenged the implicit notion of treating the brain's operations as if it was a computing device, instead emphasising its active nature and therefore how 'neural contents'conceptualisations - could not be inherently representational in nature. This led to what was termed the dynamical hypothesis (see Schacter 1996; Davids and Bennett 1998; van Gelder 1998; Freeman and Skarda 1990a, b). According to van Gelder (1998), “... a small but influential contingent of dynamicists have found the notion of representation to be 
dispensable or even a hindrance for their particular purposes" (p. 622). Schacter (1996) pointedly stated:

Could a computer engage in mental time travel, revisiting and re-experiencing the past, the way we do? ... If a computing robot cannot achieve rudimentary consciousness, then it is hard to see how it could ever engage in subjective experiences of remembering, or have the feeling that memories 'belong' to it? (p. 34).

Thus he firmly asserted that any 'cognitive software' must operate through the biological substrate of neural networks and the body within which they are grounded.

Importantly, over the last decade or so, research in neuroscience has supported such considerations, particularly those studies concerned with mental representation, for they have brought a significant and quite different bearing to what in dual-coding theory had been described as the correlations ${ }^{2}$ between images and explanations. In consequence, the theoretical underpinning to investigations involving recall — including those where semi-structured interviews are used to explore children's grasp of scientific concepts-requires a closer reexamination. An attempt is made here to accommodate what is now known about the workings of neural networks and how these affect (and effect) expressions of understanding in different modalities.

\section{Assumptions About Remembering}

As stated before, a common presumption regarding the mental representation of ideas we have acquired has been that they are entities encoded (somehow) in the cortex of the brain, entities which we access, as if drawing from a database, as and when required. In cognition research, reference has been made to them variously as concepts (see Carey 2009; Stanford 2015), mental models (see Gentner and Stevens 1983; Johnson-Laird 1983; Vosniadou and Brewer 1992, 1994) or frameworks (see Driver and Easley 1978; Driver 1981; Vosniadou et al. 2008; Vosniadou and Skopeliti 2014). They are traditionally considered to be directly elicited from episodic and semantic memory for the purpose in hand (see Tulving 1972, 2002; Wheeler et al. 1997). In this context, the term 'concepts' would include 'misconceptions' (see Gardner 1991; Smith et al. 1993), 'alternative frameworks' (Driver et al. 1985), 'prior knowledge' (Ausubel 2000), 'notions' (in particular about the Earth's shape and gravity as used in the late 1970s in the research by Nussbaum and Novak 1976 and Nussbaum 1979) and 'culturally constructed ways of knowing' (Aikenhead 1996, 1997).

Though, in developmental terms, conceptualisations change with experience and learning, the here-and-now representations we are using as we think and interact with others are traditionally thought of as having an 'intactness' or 'working' stability. It has also been considered that they are robustly independent of how we encountered the idea(s) originally, brought them into play during previous exchanges, or can act upon them in some way. That is, they have been said to be (or have become) abstract, "inherently non-perceptual, or what I will call amodal" in nature (Barsalou 1999, p. 577), somehow separate from, but useful to how we are mentally processing matters now, in the present (see also Barsalou 2003, 2008). Thus, for example, many young children who have an understanding that the Earth moves may only

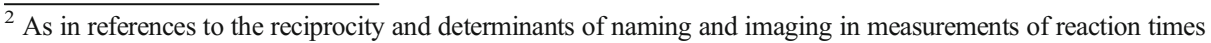
in, for example, Paivio et al. (1989).
} 
know of its orbital revolution around the Sun; they may not know of the Earth's rotational spin around its own axis (and vice versa). However, their multi-modal responses typically reveal consistency and coherence from the interviewee's perspective; the mode of representation can change but the concept or mental model is the same. In that sense, their memory of the Earth's movement is dynamic; their mental model may be well short of fuller scientific understanding and detail, but it 'works' to allow the individual to show what they make of the researcher's/ teacher's questions. Some memories of Earth, Sun and Moon (ESM) motion are also active in the sense that they involve moving imagery of rotation and revolution. They may also be dynamic in the sense of switching as children select appropriate images for the context from their repertoire of everyday and scientific concepts, some being inhibited at certain points in an interview.

Regarding mental imagery, a common presumption in much of the earlier literature in cognition was the notion that, as we try to remember, the images we are accessing are camera-like recordings of previously encountered experiences, that we are seeing again a visual image that we saw before. Researchers (in all of the inter-related fields) no longer consider this to be the case. As with recalling ideas, commonly held presumptions about visualising turn out not to be supported by the data provided in recent neuroscientific research. Before we focus on this research, it is proper to note that debates about the nature of images themselves were a preoccupation of cognitive researchers in the later decades of the last century.

\section{What We See in Our Mind's Eye}

The arguments about the nature of imagery were analysed closely in Pylyshyn's (2002) article: Mental imagery: in search of a theory, and in the 34 forum articles published in its wake (together with the author's own rejoinder). All of the papers looked at whether we should or should not adhere to the view that images are depictive phenomena akin to visual perceptions. In scrutinising relevant experimental data from the field of cognition (including those few from then available neuroscientific investigations), Pylyshyn himself considered that the evidence did not support the 'picture-theory' of images (the common-sense understanding of 'pictures in the head' or 'what we see in our mind's eye') over a 'symbol-structure' theory of mental imagery (see p. 157 in particular). He was doubtful about the nature of what was intrinsic to images. Amongst the reactions to his paper was the evidence cited by Artberry et al. (2002) which led them to reject both the literal picture-in-the-head view, and the entirely symbolic view and Bartolomeo and Chokron (2002) who concluded that space-related imagery seemed to involve both attentional and intentional aspects. Burgess (2002) considered evidence that was in favour of space-related representation, while Chatterjee (2002) pointed out that the visual cortex does not display internal visual images, though "Nestled between pictures and propositions, primitive spatial schemas with simple analog features extracted from pictorial scenes may play a subtle but wide role in cognition" (p. 186). Goldenberg (2002) suggested that imagery is "exclusively based on explicit knowledge, whereas retrieval of 'tacit' visual knowledge is bound to the presence of the object and the task of recognising it" (p. 191). In what turned out to be significant, given twenty-first century research findings about the brain's workings, Thomas (2002) noted at that time that pictures versus symbols did not exhaust the options for imagery-“embodied, active perception" (p. 211) offered an alternative. 
Moving forward to recent investigations in neuroscience, and in accord with this point, an example of how conventional presumptions do not receive support from experimental data is contained in the article by Hoenig et al. (2008) who state that:

... accumulating evidence suggests that concepts are embodied in perception and action in that their conceptual features are stored within modality-specific semantic maps in [the] sensory and motor cortex (p. 3, emphases added).

And, in their careful review of the current evidence ${ }^{3}$ concerning the structural and neural basis of conceptual memory, Kiefer and Pulvermüller (2011) conclude that "concepts are flexible, distributed representations" (p. 805).

'Distributed representation' and 'modality-specific' mean, respectively, that (i) the semantic features of each acquired concept are variously encoded by multiple layers of neural networks, not as one entity in the semantic cortex, and (ii) the networks are those concerned with sensation, expression and movement. Particular, individual networks are influenced by earlier, relevant encounters we have had, and altered by the ongoing and subsequent interchange of signals taking place between functionally separated mappings. The mechanisms described by Edelman $(2005,2006)$ as re-entry processes enable this to happen (see below).

Describing concepts as 'flexible' recognises that, contrary to common presumption, they are "tailored to the current contextual constraints, because they are constituted of dynamically recruited features depending on the context [of their recollection]" (Keifer and Pulvermüller 2011, p. 807). This emphasises the creative, as opposed to the reproductive, nature of remembering. That is, on the point of answering another's question, we put together, as best we can, (mental) entities befitting the requirements asked of us. Memory is therefore, in a very significant sense, a dynamic process, not a static reiteration. What we answer with, and how we answer, can vary depending on whether we choose, or are prompted, to respond verbally, draw pictorially, or indicate by gesture, or enact by the use of, or devising, a physical model, or might possibly result from unconscious associations (Dresp-Langley 2012). To cite Keifer and Pulvermüller (2011) again,

conceptual flexibility implies that access to a concept during language comprehension or thinking cannot be conceived as a replay of stored sensory-motor information as in a movie, but as a context-specific situation-dependent dynamic activation process (p. 809).

(See also our illustrations in respect of children's cosmologies in Bryce and Blown 2016 ${ }^{4}$ )

And, with regard to recent cognition research into imagery, it is pertinent to note the conclusion of Funk et al. (2005) (from developmental studies of children trying to position their hands to match pictures of hands photographed in a variety of postures) that their results

\footnotetext{
${ }^{3}$ Researchers draw from experimental psychological studies involving behavioural and neuroimaging trials with healthy volunteer patients, from transcranial magnetic stimulation investigations, and from case studies of patients with brain lesions in specific areas.

${ }^{4}$ We looked closely at the relationships between what was revealed as children manipulated their own playdough models and their apparent understandings of Earth/Sun/Moon concepts, focusing specifically on the switching taking place between what was said, what was drawn and what was modelled. The evidence was supportive of Edelman's view that memory is non-representational and that concepts are the outcome of perceptual mappings, a view also in accord with Barsalou's notion that concepts are simulators or skills which operate consistently across several modalities.
} 
"strongly suggest that young children's kinetic imagery is guided by motor processes, even more so than adults" (p. 402).

\section{Feedback and Re-entry Processes}

Traditional thinking about mental models (predicated on the notion of concept stability, however temporary on a longer time scale) seems so plausible and convincing. This alone may explain why it has been difficult to replace it with the view of conceptual flexibility so strongly now indicated by neuroscientific research. Perhaps, however, it has something to do with the differences between feedback and re-entry mechanisms and how the latter relate to Edelman's description of the degeneracy ${ }^{5}$ in neural circuits. Feedback mechanisms, of long-standing status, are the means through which positive reaffirmation helps to ensure the retention of our thinking. Anatomically, it seems natural to assume that the means in question involve straightforward linkages between contiguous neural circuits, that 'messages' are channelled by and within modalities, with signals returning confirmation to the semantic cortex (but see reference to Mareschal 2016 below). However, Edelman's investigations demonstrated that the brain's wiring is so much more sophisticated, its complexity ensuring multiple, near-simultaneous synchronicity between widely distributed neuronal groups. The huge number of parallel, bidirectional connections means that mental processing cannot simply be considered as the execution of algorithms rendered by straightforward feedback loops (see Edelman 2005, p. 111 in particular). Thus, when someone considers his/her answer to an interviewer's question, the individual formulates a response heavily dependent on the circumstances and may switch between a range of verbal expressions learned on earlier occasions or images constructed (or 'seen') previously (whether formally or casually). How coherent these may be will be much influenced by their previous efforts to make sense of the inter-connected meanings they have or have not forged. In relation to remembering images and explanations, recollection was implied earlier to mean 'conjointly'. We should acknowledge the interactivity or influences between them as responses are formulated-influences which are largely unconscious and possibly unknowable to the outsider. In keeping with the results of Hoenig et al. (2008, p. 18), “... conceptual features contribute to a concept to varying degrees in a flexible context-dependent manner". The individual therefore may switch between features and alter his/her contemplations mid-response. In Blown and Bryce (2017), we cite examples of children switching between everyday and scientific language during the course of interviews, thereby exposing a mixture of conceptual attributes, some of which may be in conflict (and possibly lead to confusions in the categories into which an observer might classify children's thinking). In the next section, we shall say more about 'switching'.

It is readily apparent in the literature that several writers of different persuasions find difficulty with the terms which Edelman used, some feeling it necessary to re-emphasise his findings in the face of doubt and resistance expressed by others. Interestingly, Rose considers that the term 're-entry', coined by Edelman, has itself been confusing and refers to the multiple and reciprocal interconnections amongst the module circuitry in the brain (see Rose 2006,

\footnotetext{
${ }^{5}$ Edelman used degeneracy, not to refer to deterioration, but in relation to redundancy because so many areas of the brain handle the same or similar tasks. The re-entry processes lead selectively to (self-) preferred ways of reasoning.
} 
2007). Others have been at pains to stress how different re-entry is from feedback ${ }^{6}$. Before we consider the implications of this kind of research and theorising for interview research, it is pertinent to consider a further matter, namely issues associated with investigations into conceptual change.

\section{Evidence and Argument from the Literature on Conceptual Change}

Science education researchers have, for some considerable time, adopted the constructivist perspective on learning, irrespective of the term they use for students' scientifically incorrect ideas. The literature on constructivism itself has seen debate on the how, when and what changes can be associated with students acquiring new ideas, particularly in relation to teaching methods. The mix of terms - misconceptions/alternative frameworks/preconceptions/naïve and intuitive ideas/...-is usefully considered in Leonard et al. (2014). Examples of pedagogical research publications in the science field are Rahman (2004) and Cakir (2008).

For the purposes of the present article, it is worth noting how fruitful children's cosmologies have been as an area for evidence of change and restructuring in children's thinking, beginning with the cross-cultural work of Vosniadou, Brewer and others in the 1980s (see Brewer et al. 1987). Influenced by Gentner and Stevens (1983) and Johnson-Laird (1983), they used the notion of mental models to account for the development of children's ideas and the ways in which thinking changes with life experiences and teaching. Interest in the significance of previous/early learning has grown through recent years, we ourselves having contributed to deliberations using Chinese and New Zealand children as subjects in interview research (see Bryce and Blown 2006; Blown and Bryce 2017, 2018).

Of particular relevance to matters here have been debates about 'coherence' versus 'fragmentation' in the ideas which subjects adduce during recall. In Blown and Bryce (2010), we reported on findings from multi-modal interview surveys of young people's knowledge. What children said or showed displayed coherence across modalities within and between longitudinal surveys, findings which accord with research by others, e.g. Carey (1991), Chi and Slotta (1993) and Vosniadou et al. (2008), the last of whom stated (p. 4):

At the heart of our theoretical approach is the idea that initial explanations of the physical world in naïve physics are not fragmented observations but form a coherent whole, a framework theory.

Our findings supported the position that, when asked to physically model what they thought, children evidently had some sort of mental 'content' to which they referred (sometimes explicitly by physical shape and hand positions as they formed their play-dough models). It

\footnotetext{
${ }^{6}$ For example, writing at the time of Edelman's death in 2014, Horgan, a teacher at Stevens Institute of Technology and science writer, quoted Edelman as saying in interview with him that "Re-entry is the ongoing recursive signalling between mapped areas that are made by these first few properties, so that you map maps by massively parallel reciprocal connections. It's not feedback..." (see in Horgan 2014). [According to Edelman, neural circuits develop and diversify on a Darwinian basis; neurons themselves are not the seat of memories. As Kahana (2012) has observed: “... memories are stored in the connections between neurons, and these connections enable the network to recover previously stored memories (p. 161)".] Seth, a mentee and later co-researcher of Edelman at the Neurosciences Institute in California, stated in his obituary of the Nobel laureate published in Frontiers in Psychology: "Edelman emphasized the rampantly "re-entrant" connectivity of the brain, with massively parallel bidirectional connections linking most brain regions. Uncovering the implications of reentry remains a profound challenge today" (Seth 2014).
} 
seemed likely —if we reason with either Barsalou's idea of a concept as a skill (Barsalou 2003) or Edelman's idea that “concepts are the outcome of the brain mapping its own perceptual maps leading to generalities" (Edelman 2005, p. 104) - that they were creating comparisons from mental information by means of simulations, enacted simulations. Thus, a mental model, perhaps a modification on something grasped previously, is something which is created by the individual at the point when he or she is required to recall. Consistency is reflected in similarities between past and present constructions and coherence is substantially about the interconnections amongst ideas or concepts constituting the individual's worldview. In his 'knowledge-in-pieces' theory, diSessa has a slightly different focus, arguing that coherence comes from an integration of sub-conceptual elements (his notion of phenomenological primitives, or 'p-prims') into concepts in response to specific contexts (diSessa 1983, 1988, 1993, 2008, 2017). With this focus, the initial sub-elemental ideas are incoherent and not integrated into a working schema akin to a scientific theory. It is noteworthy that Vosniadou and Brewer (1992), in an earlier study, interpreted their interview data as indicating that "there are some stable underlying conceptual structures which constrain the range of possible mental models that children can form" (pp. 575-576).

\section{The Coexistence of Everyday and Scientific Ideas}

Relevant to the discussion are the now clearer findings concerning the coexistence of everyday (or 'naïve') ideas and scientific concepts. Rather than more scientific concepts gradually replacing earlier beliefs, it may, more typically, be a matter of several manifestations continuing to exist and that what is brought to mind, or constructed on the spot, is dependent on the context of the questioning. As Driver et al. (1994) stated - in favour of the coexistence of everyday and scientific views - "Rather than successive equilibrations, it is argued that learning may be better characterized by parallel constructions relating to specific contexts" (p. 7). Others have made similar points, including Solomon (1983), Duit (1994), Taber (2000), Siegal et al. (2004), Potvin (2017) and ourselves (Bryce and Blown 2016; Blown and Bryce 2017, 2018).

Furthermore, Vosniadou (2014) has recently reported that the coexistence of naive and scientific theories is supported by two recent groups of findings from neuroscience research: (1) Fugelsang and Dunbar (2005) fMRI experiments on children's gravity concepts where Aristotelian (impetus) concepts were interfering with Newtonian ones; and (2) Shulman and Valcarcel (2012) reaction time studies verifying the truth-value of naive and scientific concepts where naive ideas were found to coexist with scientific theories. The former study on force and gravity concepts is reported more fully by Dunbar et al. (2007) and by Petitto and Dunbar (2009). Similar studies of novices and experts are reported by Brault Foisy et al. (2015) and by Masson et al. (2014). These studies reported delays in responses to questions, suggesting that coexisting images or memories or concepts were being compared. The delays were associated with activity in the regions of the brain identified with error detection such as inconsistencies in responses to questions indicating cognitive conflict as concepts compete in a process which results in dominant (more plausible) concepts being selected and less dominant (less plausible) ones being inhibited. Plausibility thus featured as a tug-of-war between everyday and scientific concepts.

Notwithstanding the tentative evidence of coexistence being revealed by neuroscience, the 'conceptual change' paradigm and the associated 'knowledge restructuring' theory, whereby naive concepts are replaced by scientific ideas as a result of development, learning and 
instruction, continues to dominate constructivist thought amongst researchers. For example, Brault Foisy et al. (2015, p. 27) firmly state that:

For Vosniadou, Ioannides, Dimitrakopoulou, \& Papademetriou, 2001, conceptual change is "a process that requires the significant reorganization of existing knowledge structures and not just their enrichment". Implicit to this, there is the idea that students' prior knowledge no longer exists after a conceptual change occurs (see Shulman and Valcarcel 2012).

However, fresh evidence from neuroscience suggests that initial concepts are suppressed by the process of inhibition but continue to function as an alternative repertoire in memory (see Mareschal 2016). These findings from traditional research methodologies and from brain research bring into question the very notion of 'conceptual change'. It seems that rather than change, naive concepts remain in memory to be recalled or re-created in response to questions set in a particular context. If the context affords everyday ideas, then naive and synthetic concepts are re-created. If the context invites scientific reasoning, then scientific concepts are re-created.

Semantic memory changes as a result of learning and episodic memory as a result of personal experience of time and place. Memory of facts and events may remain relatively intact but recollections differ as memories are re-created within specific contexts and may become blurred by 'temporal noise' with the passing of time in keeping with Edelman's (1989) 'remembered present'. Rather than a palimpsest in which the new overwrites and replaces the old, making the old almost inaccessible, both old and new memories, old (everyday/naive) and new (scientific) concepts, coexist.

In summary, children's cosmologies has proved to be a fruitful area for research into children's conceptual development: processes of conceptual change including ongoing debates concerning 'knowledge restructuring' versus 'coexistence of everyday and scientific ideas'; and 'knowledge-in-pieces' versus 'children's theories of the world'. It is also an area with the potential to build foundations in multi-media/multi-modal reasoning such as the use of models with implications for other fields of teaching and learning. As the (US) National Research Council (2007) stated:

...taking the time to construct such a robust Earth concept may be worth it for several reasons. First, it provides a foundational framework for constructing explanations of many important phenomena that connect to children's daily lives such as the reasons for day and night and the causes of the seasons. Second, it provides a wonderful opportunity for engaging in model-based reasoning during the elementary school years and developing important epistemological understandings of models (p. 105).

\section{Implications for Interview Research}

An implication apparent from the literature cited above is that mainstream notions of mental models as descriptors of children's knowledge need to be carefully reconsidered. There has been a tendency to view such models as having a degree of stability for months or even years at a time - as apparent in the categories to which many researchers, including ourselves, have 
assigned children's understandings. While children's astronomy concepts of ESM shape can be broadly categorised using mental models or astronomy concept schemes such as those developed by the authors inspired by the "Earth Notions" of Nussbaum and Novak (1976) (see Bryce and Blown 2013, Figures 1-3), these are simplifications of a rich variety of responses from children both everyday and scientific (see Blown and Bryce 2017, 2018) which can be further reduced into cosmological elements reflecting the structure of the interview (see below in this paper, Fig. 1).

Certainly, the overall patterns detectable in subject samples have helped to show how far short of a scientific grasp these understandings often are, together with the developmental changes which are likely to ensue in children exposed to the normal run of science activities in schools in those cultures where children are taught by scientifically minded teachers (see Blown and Bryce 2006 and Bryce and Blown 2007 in respect of comparisons between China and New Zealand). They afford pointers to what can be usefully focused upon by teachers in the course of instruction - particularly during those periods when significant shifts in thinking occur, such as when pupils' ideas about the solar system are changing from geocentric to heliocentric, or when animistic and anthropomorphic expressions (say, for the movement and appearances of the Sun and Moon) are all but dispensed with. (Regarding the persistence of the latter well through the primary school years, see Blown and Bryce 2018).

However, unless the ideas relating to concept flexibility and the dynamic, and possibly nonrepresentational, nature of memory are taken into account, there is the risk of researchers misrepresenting the knowledge which interviewees possess - even in the course of in-depth, semi-structured interviews. The greatest risk of underestimating knowledge will arise from mono-modal strategies, for example where only verbal statements are interpreted; the least from multi-modal strategies where crosschecks can be made via statements, drawings, models and gesture [see Bryce and Blown (2016) on verbal language, drawing, modelling and gesture; Goldin-Meadow (2017) on gesture; Anning and Ring (2005), Duffy (2006), Goodnow (1977)

Earth Shape Concept (Scientific)
Physical Shape
Spherical ${ }^{1}$
Sky synonymous with atmosphere or space ${ }^{2}$
Spherical
Ground synonymous with surface
Sky synonymous with atmosphere or space
People live all over curved surface
Understanding of gravity
Planet in Space
Member of Solar System
Note. ${ }^{1}$ The most common scientific shape was ball-like; the main everyday-cultural shape being disc-shaped (sometimes as a truncated ball).
2Drawing the ground as the surface of the Earth demonstrated the scientific view; depicting the ground as flat indicated an everyday conception.
3Describing and drawing the sky as the atmosphere surrounding the Earth, or synonymous with space was taken as the scientific view.
4Habitation of Earth and Identity with Earth were indicated by drawings of "Self" and "a friend on the other side of the Earth".
5Understanding of gravity was indicated by drawings of Self \& Friend, trajectories of thrown and dropped balls, and flow of water from bottles.
6Many older children knew that the Earth was a planet orbiting the Sun in the Solar System and knew of other planets such as Venus and Mars.

Fig. 1 Earth shape concept (scientific) 
and Malchiodi (1998) on children's drawings in general; and Vosniadou and Skopeliti (2014) on the use of children's drawings to clarify children's cosmologies]. Multi-modal methodologies require time-consuming qualitative research, increasingly difficult to tackle because of the ethical challenges now associated with one-to-one investigations with young children. Nevertheless, even with them, there are complications of interpretation with multi-modal research, for reasons discussed in the next paragraph.

The overall patterns revealed by generalising across subjects deal with the end products of their thinking, as we have argued before (Bryce and Blown 2016). They hide the fact that, at an individual level, each child's cosmology is unique and they say little about the process of concept creation. Because researchers describe what they find as end products in a representational format (influenced no doubt by the fact that that is the form of reproduction utilised in books and journals which inform and disseminate their work), they tend to take for granted a representational view of memory, a view that is not supported by the neuroscientific findings, as we have indicated here. A non-representational interpretation means that an individual, when questioned, will in fact rapidly switch between (2-d or 3 -d) images and explanations for elements potentially relevant to cues in what has been asked, formulating a response at a speed dictated by familiarity with the setting concerned and in a mode or format thought best - almost always to answer sincerely — and satisfy the questioner, perhaps to maximise status at that moment ("This is my best shot", so to speak). The elements sourced in the process will vary in accord with what relevant encounters the individual has had in the area in question. For some interviewees, pertinent images will prevail, for others they may not be available to be sourced; for some subjects, relevant words may prevail, but for others no suitable expressions may be accessible; and so forth. Crucially and for every individual, re-entry processes will mean rapid crisscrossing between and amongst elements and modalities to generate a response, whether a spoken answer, a drawing or a devised model. The mental processes taking place in the sensory and motor cortex enact/construct/re-construct thoughts, exploiting salient elements as best can. The end product is representational in nature, though others not articulated, could have been too. Neurologically, pathways involved in re-entry, whereby the same idea can be expressed through alternative modes of its demonstration, may be subject to differing degrees of degeneracy. What interviewers hear and see in the responses of their subjects is a (localised) culmination of elements shaped or dictated during the very process of their articulation. As Edelman (2006) stated: "In a selectional brain, memory, imaging, and thought itself all depend on the brain 'speaking to itself' by re-entry” (p. 57); “... memory [is] a dynamic recategorical system property” (p. 59).

\section{Summarising These Points}

To sum up at this juncture, the research literature reveals that our understandings of the expressions 'mental models', 'representational' and the 'dynamic' nature of ideas and images have all changed significantly over recent decades. Whether from a cognitive or a socio-cultural perspective, mental models in the field of children's cosmologies were largely regarded as being representational in character, even where they were said to be dynamic (with that term often not explained or defined). As representations, mental models were accorded meaning as a consequence-"a common error", according to Edelman (2005, p. 104). In effect, images and explanations were being thought of as 
remembered depictions, brought from memory and put into play fully formed as meaningful concepts (meaningful in the sense grasped by the interviewee). Now, we can interpret them as enactments, constructed - at the point of recall-from elements (cf. diSessa's 'p-prims'). Recent findings, especially the insights from brain research, have enabled us to recognise the complexity of the recollection processes; how these in themselves are possibly non-representational; but where their products are of such a nature, with all that that implies for interpreting what the interviewee seems to know (i.e. non-representational processes may produce representational products).

The dynamism lies in their construction during the creative act of recall, not in a variety of replications being in contest with each other for application, nor as a single mental depiction possessing some special property rendering it capable of varied expression. The psychological and neurological perspectives, exemplified by Barsalou and Edelman, respectively, suggest avenues for research, though neither seems easily operationalised or straightforwardly put into testable hypotheses, by virtue of the creative component of remembering. We can reason in accord with Barsalou (interpreting concepts as situated simulations or skills in creating images consistently over a range of stimuli and responses) or in accord with Edelman (recognising that concepts are generated by re-entry processes involving a variety of neural pathways, the substrate of which does not carry representations). Either way, dynamicism achieves the 'fuzziness' rather than the 'digitality' which Davids and Bennett (1998) stressed is the nature of human remembering. Memory is in a continual state of flux, being constantly updated. It is regenerated each time an internal or external cue triggers a conscious reflection on present or past events. Such cues can be personal musings or questions asked during an interview; and the associated reflections often involve imagery. Thus imagery and memory are features of consciousness itself. As Edelman observes:

We experience primary consciousness as a 'picture' or a 'mental image' of ongoing categorized events. But ... there is no actual image or sketch in the brain. The 'image' is a correlation between different kinds of categorizations... Primary consciousness is a kind of 'remembered present' (Edelman 2001, p. 119).

We note that Pylyshyn's symbol theory is framed by the paradigm that memory is representational. However, memory may be non-representational as advocated by Edelman, with information resulting from the interconnections in networks. Non-representational memory is not necessarily unconscious. When a concept is re-created in response to a question, the response is both conscious (in the sense that we are deliberately responding to questions about a concept) and unconscious (in the sense that we don't know the processes of re-creation of the concept). From the research perspective, the investigator's task is to consider alternative interpretations regarding interviewees' statements about what they see in their 'mind's eye'.

\section{Research Question}

Acknowledging the differences in how interviewees respond during in-depth, multi-modal investigations; the not insignificant extent of mode switching (see Bryce and Blown 2016 and Blown and Bryce 2017); and the challenge posed by the considerations concerning 
mental construction and representation set out earlier, we scrutinised a wide range of interview protocols for evidence as to:

- (a) Whether and when children were referring to images linked to their answers and explanations (spontaneously or cued)

- (b) Whether or not images related to previous recollections in those cases where children were re-interviewed after a passage of time

- (c) The extent of relevant explanations provided by children where they reported no associated imagery.

Formalising these as a research question:

RQ What insights into imagery are possible from children's concepts of the Earth, Sun and Moon?

\section{Methodology and Participants}

\section{An Empirical Study}

As in our earlier research, the methodology used here utilised verbal language, drawing and play-dough modelling to obtain multi-modal responses to questions from an extensive interview guide (see Blown and Bryce 2013, Appendix G) by means of Piagetian style interviews and Socratic dialogue (Piaget 1929, 1930). Full details have been described in our previous publications concerning children's knowledge restructuring in this area (Blown and Bryce 2006), the coherence detectable in their conceptualisations (Blown and Bryce 2010), comparisons between novices and experts (Bryce and Blown 2012) and children's grasp of the shape and size of the ESM (Bryce and Blown 2013). Children were asked about the motion of the Earth, the apparent motion of the Sun while watching the changes in the shadow of a shadow stick and the motion of the Moon through observation. The motion studies included associated concepts of time; Daytime and Night-time. The children then drew and modelled the shape and motion of the ESM and explored the concepts of 'Habitation of Earth' and 'Identity with Earth' by drawing and modelling themselves and 'a Friend who lived on the other side of the world'. This was followed by them modelling the motion of the ESM; and Daytime and Night-time using their own play-dough models. Next, children studied concepts of gravity through a series of thought-experiments involving throwing and dropping balls, and studying the action of water/juice in drink bottles on this side and the other side of the Earth; and dropping a ball into a hole through the Earth. Finally, children studied seasons and eclipses (see Blown and Bryce 2010, 2013). At the end of their interview, children were asked the following questions about imagery: (1) When I asked you about the Earth, Sun and Moon did you think of any stories you have been told about them? (2) Did you see any words (in your imagination)? (3) Did you see any pictures (in your imagination)? (4) Did you see anything moving (in your imagination)? (5) Where did your ideas come from? (6) What were you thinking of? The whole interview based on the interview guide lasted up to an hour or so depending on the attention span of the child, but was broken into shorter sections for drawing and modelling. Our previous research has consistently shown that young children's cognitive 


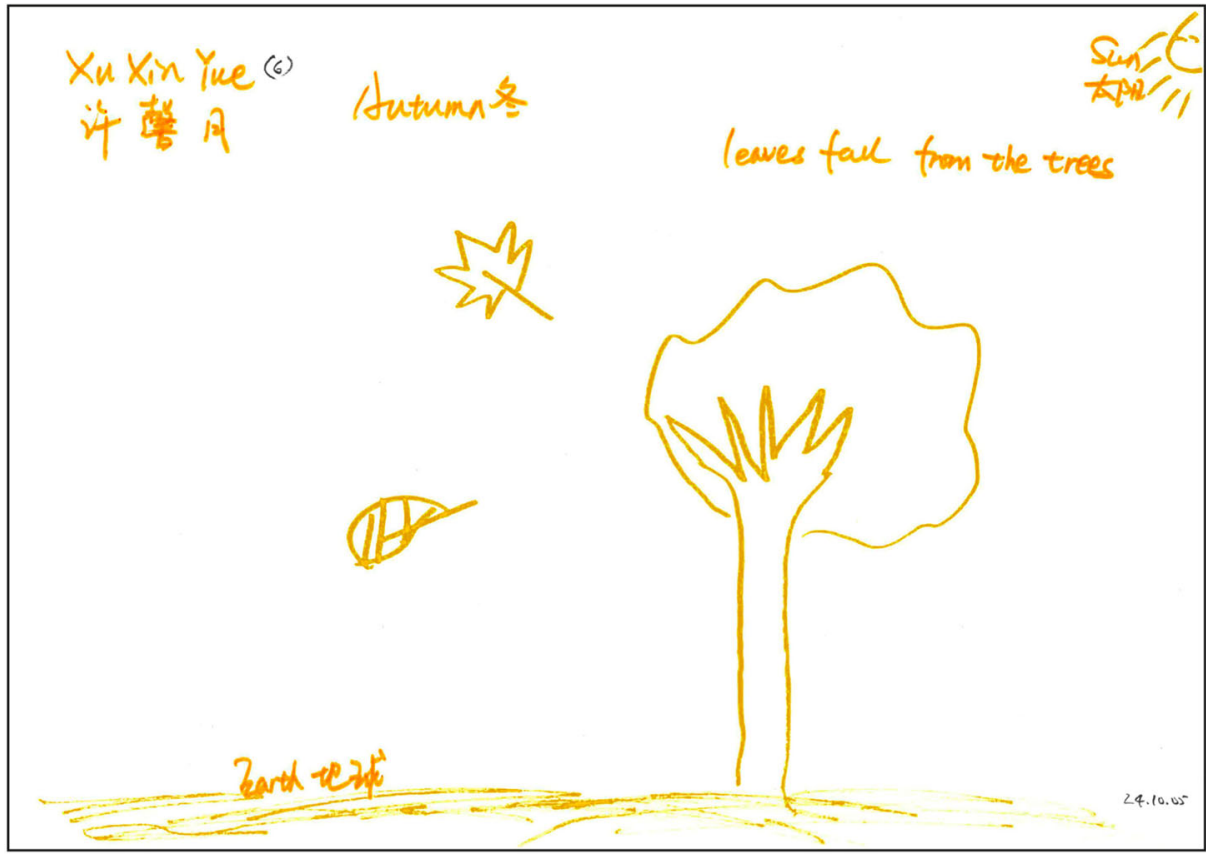

Interview Protocol

R. Why do the seasons change?

C. Because of the changes of the Earth.

$\boldsymbol{R}$ What season is it now?

C. Autumn.

R. Draw Autumn.

C. (Draws Earth in Autumn).

$R$. Are these leaves falling off the trees?

C. Yes.
Imagery Protocol

R. When I ask you about the Earth and the Sun and the Moon can you see any pictures in your imagination?

C. Yes.

$R$. Can you see anything moving in your mind?

C. No.

R. When you look at this drawing here (I indicate child's drawing of the seasons) can you imagine leaves falling from the trees?

C. Yes.

Fig. $2 \mathrm{Xu}$ drew a separate drawing for each Season and believed that the Sun was closer to the Earth in summer. $\mathrm{Xu}$ could not imagine ESM moving but could visualise leaves falling from trees in Autumn.

abilities have been underestimated and they are quite capable of introspection and metacognition (see Donaldson 1978, Gascoine, Higgins and Wall 2016). Initial questions put to children may be considered novel (having in all probability never been asked such questions before); hence, the follow-up questions allowed the situation to be clarified.

\section{Participants}

One hundred and forty-one children (age 3-12) took part in the study: 73 from Wairarapa, Wellington Province, New Zealand (36 boys and 37 girls), and 68 from Changchun, Jilin Province, North East China (34 boys and 34 girls), with the subjects being matched on socio-economic background based on parents' occupations. There 


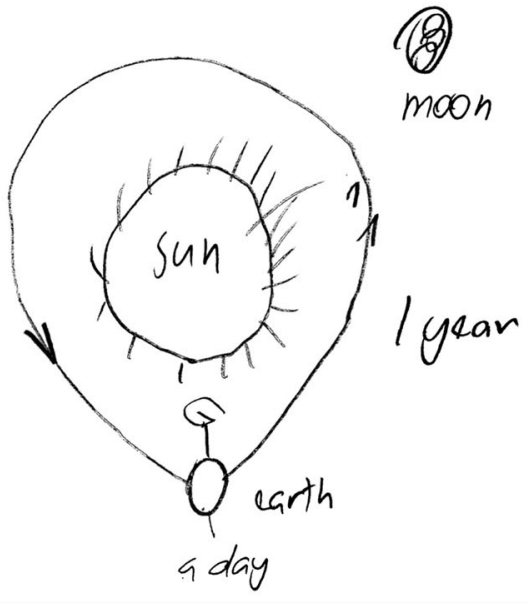

\section{Interview Protocol}

R. Can you tell me about the Sun?

C. It lights up the planets and it's hot as (very hot) and it's in space and even if I'm miles and miles away it's still very hot in there.

R. Don't look at the Sun-but where is the Sun'

C. In space.

R. Is the Sun moving?

C. Yes - it moves.

R. How does it move?

C. It just-Oh-it doesn't move planet revolves around the Sun.

R. The planet - the Earth - goes around the Sun?

C. Yes - the Sun doesn't move.

[Tekaranga drew the Earth rotating in a day and revolving around the Sun in a year.]

\section{Imagery Protocol}

R. When I asked you about the Earth and the Sun and the Moon did you think of any stories you had been told about them?

C. Yes - "The cow jumped over the Moon" that's about as much as I can remember.

R. What about the story of "Maui and the Sun" do you remember that?

R. II remind child of a children's book on a Māori Legend (Maui and the Sun by Bishop, 1996).

C. Yes - he caught the Sun.

R. He slowed the Sun down, didn't he?

C. Yes - he slowed it down because it went too fast

R. With ropes?

C. Yes - with ropes.

$R$. When you think of these stories do you see any words or any pictures in your imagination?

C. No-not really.

$R$. When you talk about things spinning - do you imagine them spinning?

C. Yes.

R. Can you see them in your mind, or not?

C. Yes - I can see them in mv mind-sninning.

Fig. 3 In the story Maui and Sun, the Sun is described as moving around the Earth too quikly: Maui captures it with ropes and slows it down. Tekaranga thought that the Earth rotated and revolved around the Sun. His memory thus has both everyday and scientific imagery.

were 47 kindergarten and pre-school children age 3-5 (NZ 24, China 23), 55 junior primary school children age 6-8 (NZ 28, China 27) and 39 senior primary school children age 9-12 (NZ 21, China 18). 
Table 1 Details of responses to mental imagery questions

Specific: Pictures of Earth, Sun and Moon.

\section{Static Imagery}

General: Images of Self and new Friend who lives a long way away; and images of a Well-hole

\begin{tabular}{|c|c|c|c|c|c|c|}
\hline \multirow[t]{3}{*}{ Group } & \multicolumn{4}{|c|}{ General } & \multirow{2}{*}{\multicolumn{2}{|c|}{$\frac{\text { Specific }}{\text { Pictures ESM }}$}} \\
\hline & \multicolumn{2}{|c|}{ Self \& Friend } & \multicolumn{2}{|c|}{ Well-hole } & & \\
\hline & $\overline{f / n}$ & $\%$ & $f / n$ & $\%$ & $\overline{f / n^{*}}$ & $\%$ \\
\hline NZ Kindy \& Pre-School & $18 / 22$ & 82 & $7 / 22$ & 32 & $4 / 9$ & 44 \\
\hline China Kindy \& Pre-School & $13 / 16$ & 81 & $8 / 16$ & 50 & $3 / 10$ & 30 \\
\hline NZ Junior Primary School & $20 / 21$ & 95 & $18 / 21$ & 86 & $7 / 12$ & 58 \\
\hline China Junior Primary School & $22 / 22$ & 100 & $20 / 22$ & 91 & $13 / 22$ & 59 \\
\hline NZ Senior Primary School & $23 / 23$ & 100 & $23 / 23$ & 100 & $11 / 17$ & 65 \\
\hline China Senior Primary School & $22 / 22$ & 100 & $21 / 22$ & 95 & $14 / 22$ & 64 \\
\hline
\end{tabular}

*Only those children who had concepts of the Earth, Sun and Moon were asked specific questions.

Dynamic Imagery

Specific: Images of the Motion of the Earth, Sun and Moon

General: Images of the motion of dropped and thrown balls; drink falling from or staying put in a bottle on the other side of the Earth; a ball falling through a hole through the Earth

\begin{tabular}{|c|c|c|c|c|c|c|c|c|}
\hline \multirow[t]{3}{*}{ Group } & \multicolumn{6}{|c|}{ General } & \multirow{2}{*}{\multicolumn{2}{|c|}{$\begin{array}{c}\text { Specific } \\
\text { Motion ESM }\end{array}$}} \\
\hline & \multicolumn{2}{|c|}{ Balls } & \multicolumn{2}{|c|}{ Bottles } & \multicolumn{2}{|c|}{ Hole-Through } & & \\
\hline & $f / n$ & $\%$ & $f / n$ & $\%$ & $f / n$ & $\%$ & $f / n^{*}$ & $\%$ \\
\hline NZ Kindy \& Pre-School & $21 / 22$ & 95 & $7 / 22$ & 32 & $8 / 22$ & 36 & $4 / 9$ & 44 \\
\hline China Kindy \& Pre-School & $15 / 16$ & 94 & $5 / 16$ & 31 & $5 / 16$ & 31 & $3 / 10$ & 30 \\
\hline NZ Junior Primary School & $21 / 21$ & 100 & $12 / 21$ & 57 & $15 / 21$ & 71 & $6 / 12$ & 50 \\
\hline China Junior Primary School & $22 / 22$ & 100 & $13 / 22$ & 59 & $14 / 22$ & 64 & $13 / 22$ & 59 \\
\hline NZ Senior Primary School & $23 / 23$ & 100 & $21 / 23$ & 91 & $21 / 23$ & 91 & $12 / 17$ & 71 \\
\hline China Senior Primary School & $22 / 22$ & 100 & $20 / 22$ & 91 & $17 / 22$ & 95 & $17 / 22$ & 77 \\
\hline
\end{tabular}

*Only those children who had a spherical Earth concept and a knowledge of "other countries" and/or the concept of "the other side of the Earth" were asked about Bottles and Hole-through. Only those children who held concepts of the Earth, Sun and Moon were asked specific questions.

Note. There were no significant differences between the cultures apart from greater knowledge of well-holes on the part of children in China (where wells are more common than in NZ). Both Static and Dynamic imagery can be seen to increase with age as a result of development and experience with the world including learning from teachers, parents, books, TV and other knowledge sources. 


\section{Data Collection, Analysis and Coding}

The interviews were recorded on audio and videotape and transcribed by the researcher (with the assistance of interpreters in China). These protocols together with children's drawings and models provided the main qualitative evidence informing the RQ. The interview protocols (see left-hand columns of Figs. 2 and 3) together with children's drawings and models were initially analysed and coded by the researcher using the Cosmological Concept Categorisation Scheme developed by the authors (see Bryce and Blown 2006). This was used to identify examples of non-spontaneous static imagery.

These included specific static images - such as pictures of ESM and general static images - such as images of Self and a Friend who lives a long way away (on the other side of the Earth) and images of a well hole or rabbit hole (as in Lewis Carroll's Alice-in-Wonderland) - as well as dynamic imagery such as "imagining a ball falling through a hole through the Earth". A range of exemplars from each category were checked by two astronomy educators ${ }^{7}$ from Carter Observatory in Wellington, NZ, with an inter-coder agreement of 85-95\%; Cohen's kappa $\kappa=0.83$ to 0.94 . The imagery protocols (right-hand columns of Figs. 2 and 3) captured examples of imagery in response to specific questions, were grouped into categories by the researcher and his interpreters and were subject to percentage analysis as in Table 1 .

\section{Results: Insights into Imagery Afforded by the Studies}

The methodology afforded insights into a variety of forms of imagery depending on the manner of questioning or the nature of the dialogue. The core questions remained constant by the use of the interview guide; Socratic dialogue served to supplement the standard questions. Although some results were 'generalised' for quantitative analysis, the qualitative richness of responses is evident from the protocols. Importantly and wherever possible, the interview questions were triangulated using verbal language, drawing and modelling modalities. Examples of drawings are included as figures.

\section{Spontaneous (Familiar Context, Concept-Cued) Imagery}

In response to the questions about the shape and motion of the ESM:

Did you see any pictures (in your imagination)?

Did you see anything moving (in your imagination)?

Many children were able to visualise the shape and motion of ESM but sometimes the imagery was of everyday concepts rather than the scientific interpretation. They positively affirmed that they could see the Earth rotating and revolving when asked.

\footnotetext{
${ }^{7}$ Because the research focuses on children's astronomy concepts and associated imagery, we selected independent assessors who had a background in astronomy and teaching to check our categories. The external coders were both experienced astronomy educators who were active astronomers and teachers who taught astronomy to visiting groups of adults and children at Carter Observatory, to student teachers at Victoria University of Wellington Teachers College and Faculty of Education and to teachers and children at local schools. To maintain consistency and reliability, we have used the same external coders throughout our studies.
} 


\section{Protocols}

Aroha (NZ Māori: female; age 7 years 0 months)

$R$. When I asked you about the Earth, Sun and Moon did you think of any stories you have been told about them?

C. No.

$R$. Did you see any words or any pictures (in your imagination)?

C. I've seen one picture.

R. In your imagination?

C. Yes.

$R$. What's the one picture you saw then?

C. I've seen a Moon - it's a full-Moon - and all the dogs howl!

Samantha (NZ European: female; age 8 years 8 months)

R. When I asked you about the Earth and the Sun and the Moon did you think of any stories that you have been told about them?

C. No.

R. Did you see any pictures or think of any pictures?

C. Yes.

R. What pictures?

C. Planets - in a book - with pictures in it - I was just looking - I wasn't thinking about the book - (the) story, words (or) writing - I was just thinking about the pictures in it.

$R$. Could you imagine anything moving?

C. Yes - the Sun and the Earth.

Deng Chu Heng (China: Han: male; age 6 years 4 months)

$R$. When we talk about these things - about the seasons and about the Earth and the Sun and the Moon, can you imagine them in your mind?

C. Yes.

R. Can you imagine things moving?

C. No.

$R$. But you know they are moving?

C. I have no pictures of the Earth, Sun and Moon moving but I can imagine the leaves and the snow falling.

Zhang Zhe (China: Han: male; age 8 years 10 months)

$R$. When you talk about things moving, can you see them moving in your imagination?

C. Yes.

R. When you're talking about eclipses and things like that, can you see pictures in your imagination?

C. Yes.

R. Do you see any words in your imagination?

C. No. 
Zhou Yi Ping (China: Han: female; age 9 years 3 months)

$R$. When we talk about the seasons and about the Earth and the Sun and the Moon, do you get any pictures in your imagination?

C. Yes.

$R$. Can you imagine things moving?

C. Yes.

\section{Non-spontaneous (Unfamiliar Context, Dialogue-Cued) Imagery}

These were cued by dialogue such as:

Imagine that you're going to draw yourself on the Earth...

Imagine that you've got a friend who lives in (China, New Zealand) on the other side of the Earth...draw your friend...place the model of your friend on the play-dough model of the Earth.

Most primary children could recall or re-create mental pictures of the shape of the ESM; or imagine a friend or relative who lives on the other side of the Earth (even where their concept of it was of the 'dual-hemisphere' variety) (see Bryce and Blown 2013, Figure 1; Categories 7 $\& 8$ ), or imagine a well hole (or wishing well). They did so without difficulty but their ability to place themselves and their friends on the Earth scientifically depended on their understanding of the shape of the Earth and their concepts of gravity. In this case, imagery was inferred from children's drawings and models (such as Friend on the other side of the Earth), but there were examples of dialogue resulting in imagery of unfamiliar contexts and one capturing a child in the act of imagining.

\section{Protocols}

Ji Jing Xian (China: Han: female; age 10 years 2 months)

$R$. When you draw the seasons and you think about the Earth and the Sun and the Moon and you show things moving, can you imagine things moving?

C. Yes.

R. Do you have pictures in your imagination?

C. Yes.

R. Like - could you imagine the hammock swinging?

C. Yes.

[Child had drawn a person swinging in a hammock in summer.]

Lu Xing Yu (China: Han: male; age 9 years 8 months)

R. When we're doing things like this (modelling eclipses) can you imagine things moving in your mind?

C. (Closes eyes to imagine) - Yes! 


\section{Thought-Experiment (Dialogue-Cued) Imagery}

Imagination is thought to play a crucial role in thought-experiments, particularly those used to probe children's intuitive concepts of gravity. This was cued by questions about the motion of falling objects such as thrown and dropped balls, water or juice staying put or flowing from a bottle on this side and the other side of the Earth, and a ball falling through a hole through the Earth as follows:

Imagine that you throw a ball up into the air; what happens to the ball?

Imagine that you have a drink of orange juice from a bottle. You don't drink all of it, there is some left. Imagine that you put the bottle down on the ground carefully with the top off: what happens to the juice?

Imagine that your friend on the other side of the Earth puts their drink bottle down on the ground-what happens to their juice?

Imagine that there is a well hole in your garden that is very, very deep. Imagine that you dropped a ball into the well hole: what happens to the ball?

They could also imagine seasonal change such as falling leaves in autumn and falling snow in winter. However, sometimes imagery cases were difficult to classify because they were intertwined, as when children could imagine seasonal change but not ESM motion. It was evident that in some cases general imagery was associated with more common phenomena such as seasonal effects rather than the less tangible phenomena of specific imagery such as the rotation and revolution of the Earth. It is also possible that a child's drawing of the seasons leaves an image in memory which is more accessible than images associated with drawing of the motion of the ESM which was done earlier in the interview, the imagery questions being usually asked immediately following questions on the seasons.

\section{Protocols}

Natasha (NZ European: female; age 10 years 8 months)

$R$. When I asked you about the Earth and the Sun and the Moon did you think of any stories that you've been told about them or stories you've read about them?

C. (Shakes head).

R. Did you see any pictures in your imagination - like when I was telling you about Alice falling down that hole - could you imagine that?

$R$. Or the ball falling down the hole?

C. (Nods).

R. You could imagine that?

C. (Nods).

R. And when I was asking you about the Earth and the Sun and the Moon moving, could you see them moving in your imagination - or not?

C. (Nods).

R. [I clarify that a shake of the head means "No" and a nod means "Yes"]. 
Jiang Ting Yu (China: Han: female; age 5 years 10 months)

$R$. When we talk about the Earth, and the Sun and the Moon and things like the ball falling down the hole, can you imagine things moving?

Li Jin Zhu (China: Han: female; age 5 years 9 months)

R. When we talk about the Earth and the Sun and the Moon and things moving (and) we talk about the seasons and the swallows flying and things like that - and the leaves falling from the trees and the snow falling - can you imagine things moving in your imagination?

C. Yes.

R. Can you imagine the swallows?

C. Yes.

$R$. Can you imagine the swallows moving?

C. Yes.

Wang Pu Yi (China: Han: male; age 7 years 7 months)

R. When we talk about the Earth and the Sun and the Moon and the seasons, can you imagine things moving?

C. Yes.

R. When you were drawing the snow or the leaves can you imagine them falling?

C. Yes.

Zhu Xing Yu (China: Han: male; age 8 years 0 months)

[Child drew falling leaves and flying beetles as characteristic of autumn].

R. When you imagine (draw and talk about) things like leaves falling and beetles flying can you actually see them flying or falling in your imagination?

C. Yes.

R. And when we talk about the Earth and the Sun and the Moon moving, can you imagine them moving?

C. Yes.

\section{Story-Related Imagery Involving Recollections from Books, Videos and the Internet}

In response to the questions:

When I asked you about the Earth, Sun and Moon, did you think of any stories you have been told about them? Did you see any words (in your imagination)?

Young people recalled or re-created dynamic imagery of the ESM from illustrations in books such as children in New Zealand imagining the Māori hero Maui capturing and slowing the Sun with ropes in Maui and the Sun (Bishop 1996). Some could recall words in the form of rhymes from the book Alice in Wonderland (Carroll 1865/2003; on which the hole-throughthe-Earth thought-experiment is based), but they could not visualise the words as such. Others 
could visualise sections of the video of the story. In other words, children could recall specific spoken words in a musical or rhythmical form (such as rhyme) but not general dialogue. Children in China could recall stories of the creation of the Sun and Moon from 365 Nights stories (Zhang 2011).

\section{Protocols}

Joshua (NZ European: male; age 9 years 6 months)

$R$. When I asked you about the Earth, Sun and Moon, did you think of any stories you have been told about them?

C. Yes.

R. Have you ever been told any stories about the Earth and the Sun and the Moon?

C. I've been told three.

R. What were the stories?

C. I can't remember the names - two of them were Māori ones - one of them was - there were a couple of people (Maui) who were trying to stop the Sun from moving so fast.

Zoya (NZ European: female; age 8 years 7 months)

[Extract from "Hole through the Earth" thought experiment.]

$R$. Imagine what would happen to a ball dropped into a hole through the Earth. I got this idea from the story Alice in Wonderland. Do you know the story?

C. Yes.

R. What happened then?

C. She fell down into the little room ... and the rabbit went into the door (entrance to the rabbit hole) and said "I'm late, I'm late, for a very important date."

Child chants verse to tune. Sequence of events is jumbled but essence of story is intact.

Zoe (NZ European: female; age 8 years 4 months)

R. When I asked you about the Earth, Sun and Moon, did you think of any stories you have been told about them?

C. Yes.

R. Did you see any words or pictures (in your imagination)?

C. Yes.

R. Did you see anything moving in your imagination?

C. Yes.

R. Where did you get your ideas from?

C. From the book (Alice in Wonderland).

$R$. When I asked you about the hole going through the Earth what were you thinking about?

C. I was thinking about a video of it.

R. Have you seen a movie of Alice - a video?

C. Yes. 
Daniel (NZ European: male; age 9 years 7 months)

$R$. When I asked you about the Earth, Sun and Moon did you think of any stories you have been told about them?

C. No - not really - but I've read lots of books about the Earth and that moving around.

R. Did you see any words or any pictures or anything like that when you were thinking about the Earth and the Sun and the Moon?

C. Yes I do - I think about it moving around and see it.

R. So you see it as a kind of picture?

$\boldsymbol{R}$. When you think about it - when you see pictures of things - or visions of things can you see them moving?

C. Yes.

R. In your mind?

C. Yes - in my mind - when I think - talk about things moving - I think about them moving.

Tekaranga (NZ Māori: male; age 9 years 7 months)

Recalled "The cow jumped over the Moon" and the Māori legend "Maui and the Sun"(see Fig. 3).

Yang Feng Xu (China: Han: male; age 9 years 4 months)

$R$. When I asked you about the Earth, Sun and Moon did you think of any stories you have been told about them?

C. Yes (child relates story of Sun from "365 nights")

$R$. Did you see any pictures in your imagination?

C. Yes (continues story of Sun from "365 nights")

R. Do you see things moving when you think of these stories - do you see them moving in your imagination?

C. Yes.

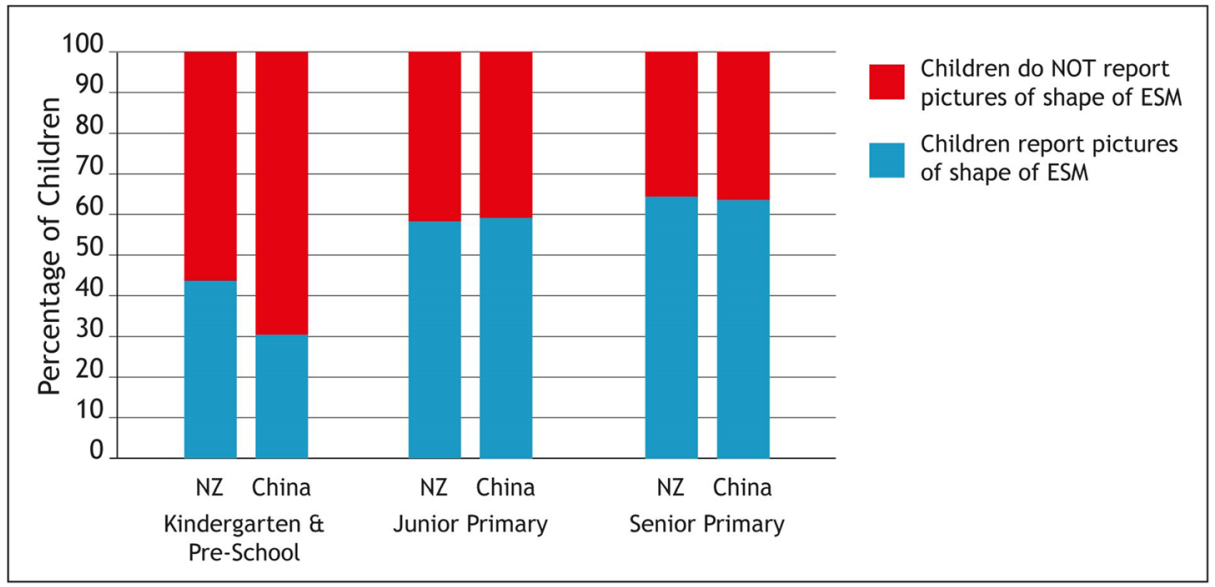

Fig. 4 Percentage of children reporting/not reporting pictures of shape of ESM in their imagination 


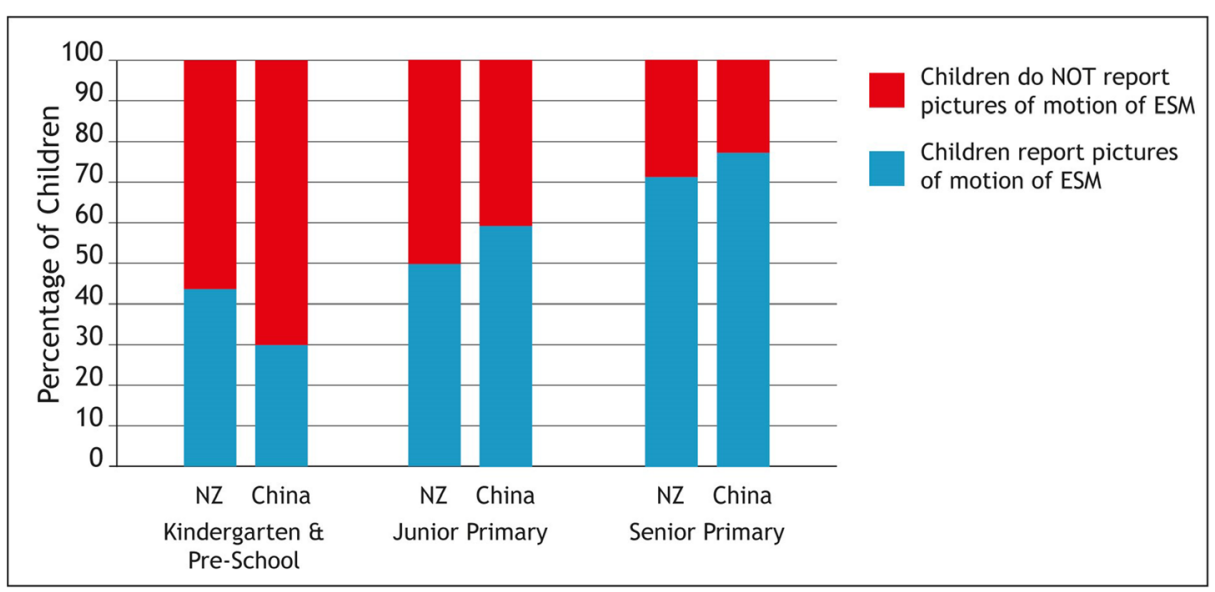

Fig. 5 Percentage of children reporting/not reporting pictures of motion of ESM in their imagination

When I ask you questions about the play-dough and so on, can you imagine things moving?

C. Yes.

\section{Additional Forms of Imagery}

In addition to these four predicted types of imagery, three additional forms were found: those inspired by (a) visits to museums, (b) dreams and (c) unconscious imagery becoming conscious through Socratic dialogue focusing on what the child had said, drawn or modelled. Sometimes concepts such as the motion of ESM could be recalled and the source of information identified, but the child had no knowledge of words or pictures or imagery.

\section{Imagination Without Pictures or Imagery}

As described above, at the end of their multi-modal interviews, children were asked whether they could see pictures or images when they were thinking about the shape and motion of ESM, habitation of and identity with Earth; intuitive concepts of gravity; and seasons and eclipses. Examples of the interview protocols, imagery protocols, Socratic dialogue and drawings are given in Figs. 2 and 3. Regarding Fig. 3, the Imagery protocol shows Socratic dialogue where the researcher probes what the child knows about the legend of the Maori hero Maui slowing the Sun. In the legend at the foot of Fig. 3, 'everyday' imagery (in contrast to 'scientific' imagery) refers to the now conventionally understood meaning as 'naïve', 'intuitive' or 'non-scientific'.

The percentages of children reporting that they see pictures of shape, and dynamic-imagery of the motion, of the ESM in their imagination at three school stages (kindergarten and preschool, junior primary and senior primary) are displayed in Figs. 4 and 5. They show an increase in reported pictures and imagery with age. However, almost all of these children demonstrated that they could imagine (picture) static concepts such as: 


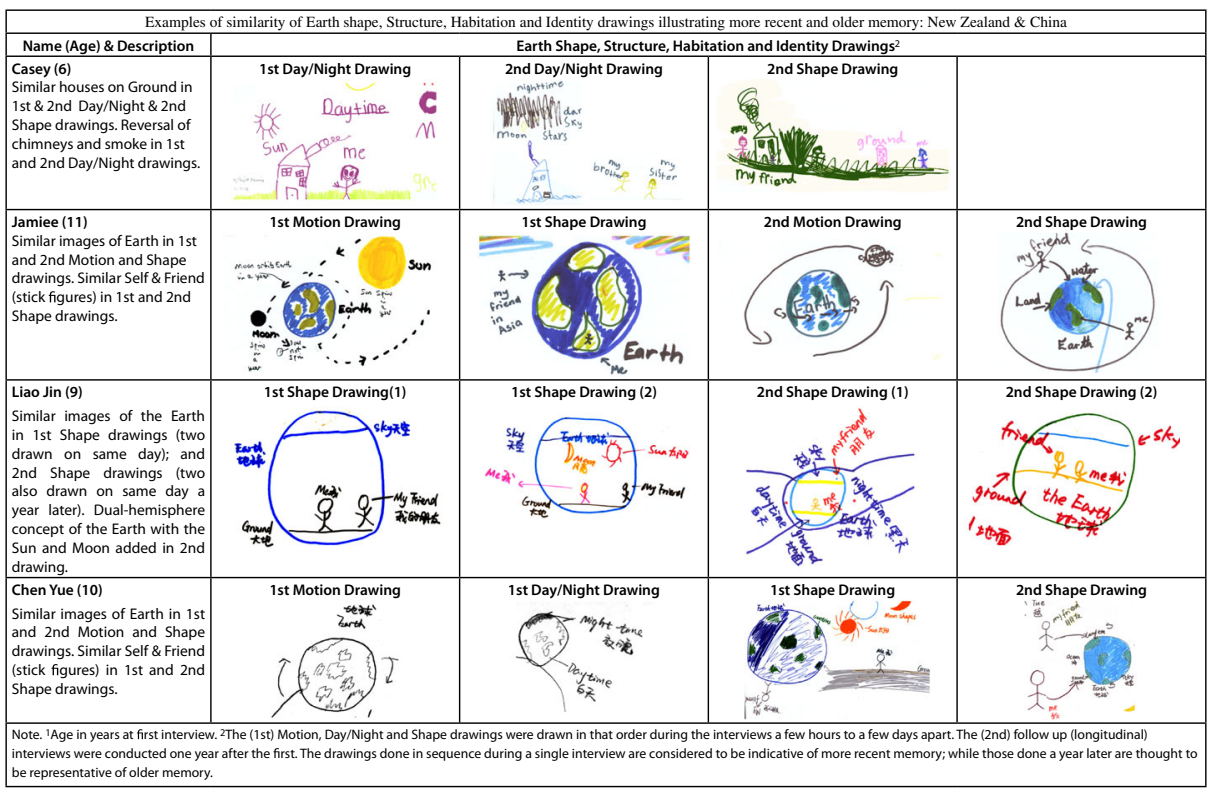

Fig. 6 Examples of similarity of Earth shape, structure, habitation and identity drawings illustrating more recent and older memory: New Zealand and China

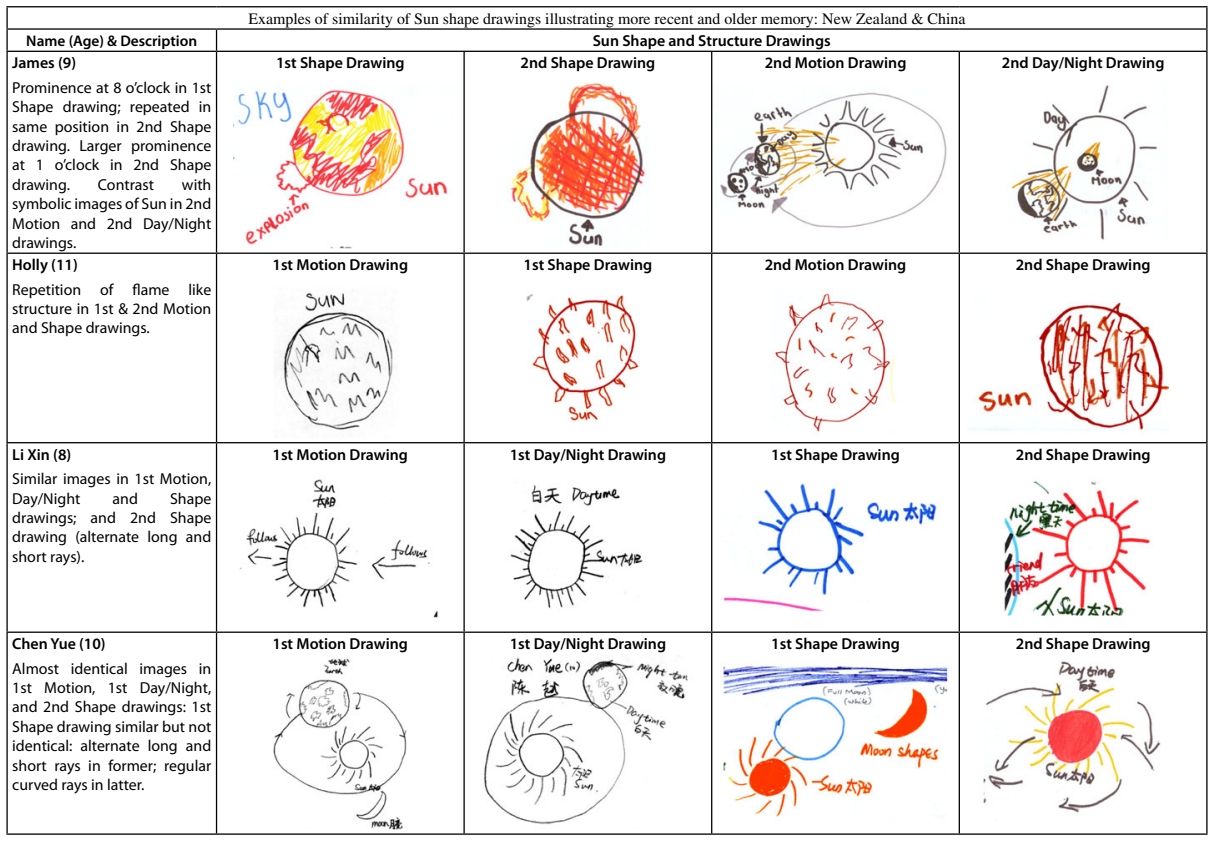

Fig. 7 Examples of similarity of Sun shape drawings illustrating more recent and older memory: New Zealand and China 


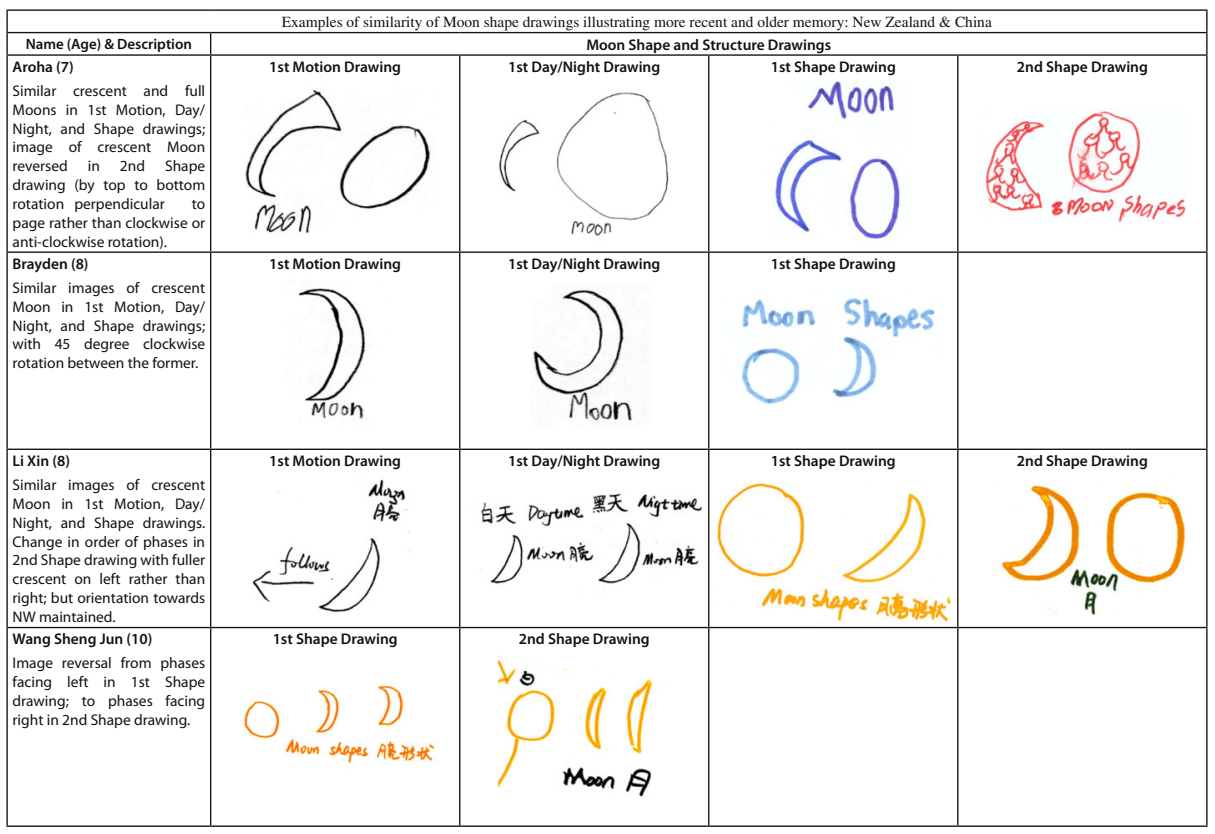

Fig. 8 Examples of similarity of Moon shape drawings illustrating more recent and older memory: New Zealand and China

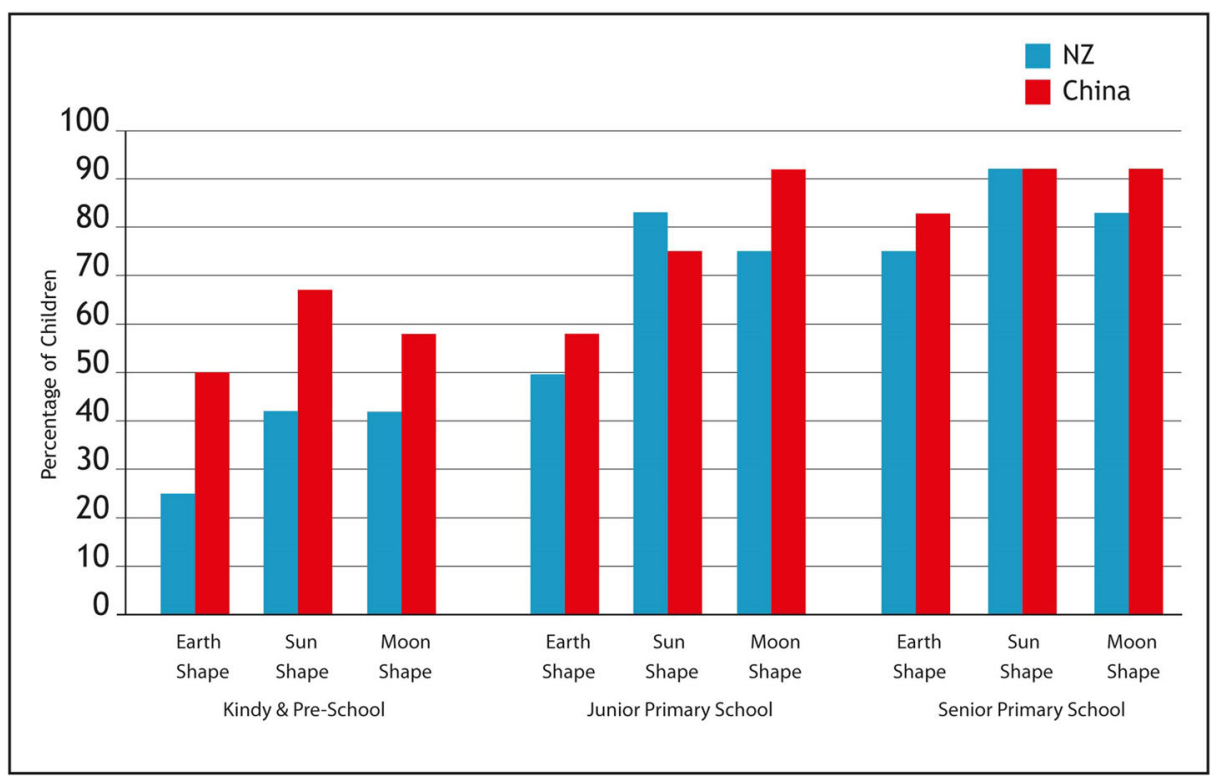

Fig. 9 Frequency of similar images in the same interview (more recent memory) 


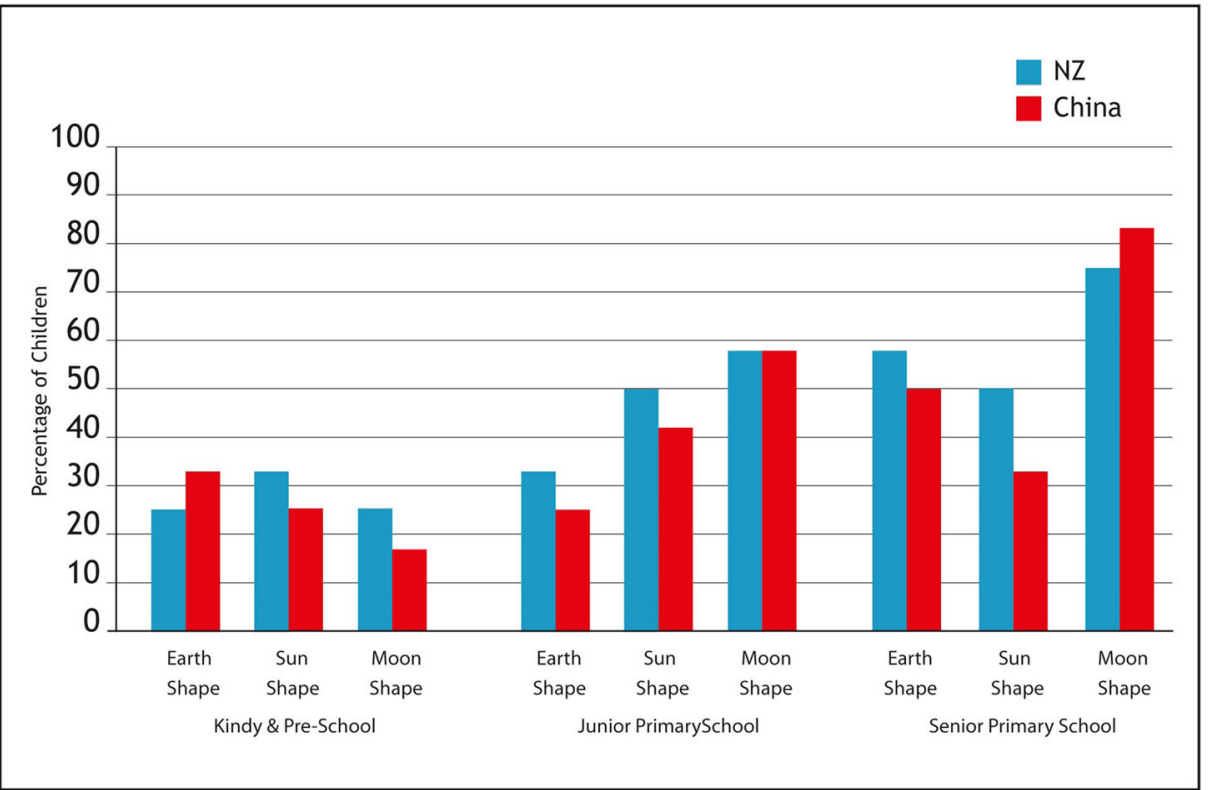

Fig. 10 Frequency of similar images in longitudinal repeated interview (older memory)

- The shape of ESM

- Themselves living on this side of the Earth (habitation and identity study)

- Friends living a long way away on the other side of the Earth (habitation study)

- Well holes (preliminary to hole-through-the-Earth thought-experiment)

(see Bryce and Blown 2013)

They could also imagine dynamic concepts such as:

- The motion of ESM

- Throwing and dropping balls; water/juice falling from a bottle on this side and the other side of the Earth

- A ball falling through a hole through the Earth (as part of gravity studies)

(see Blown and Bryce 2013, data in Table 1)

It is worth noting that there were no significant differences between the cultures apart from greater knowledge of well holes on the part of children in China (where wells are more common than in NZ). Both static and dynamic imagery can be seen to increase with age as a result of development and experience with the world including learning from teachers, parents, books, TV and other knowledge sources. The positive responses showed a coexistence of everyday and scientific ideas, and imagination was generally found to be active throughout the interviews, sometimes tending to take the subject matter off on a tangent as children shared their experiences and offered their hypotheses of phenomena. Looking at the results another way: If such high percentages of NZ and China children report a lack of pictures of ESM shape and a lack of imagery of ESM motion, how can they actually create and re-create concepts of ESM shape and motion in three different modalities? Considering the implications for or 
against a representational form of memory, these results do appear to suggest that children have two forms of imagination: one conscious in the form of 'pictures in the mind' or imagery that can be created by mental processes prompted by questions as in an interview setting and shared with others through dialogue, drawing and modelling. The second unconscious imaginings involving non-representational processes with no conscious pictures or imagery, but nevertheless able to create and re-create static and dynamic concepts such as the shape and motion of ESM - the conscious form of imagination becoming more common with age; the unconscious form being general.

\section{Similarity of Representations of Imagery over Time}

It was found that there were similarities between ESM shape and motion drawings over time. Although the process is unknown, it seems that the mind is capable of re-creating identical or almost identical images over time and these can be represented as drawings. The minor differences between drawings suggest either slight differences in the process of image creation or slight errors in the process of translation of the images into drawings through representation (see Figs. 6, 7 and 8, where the thumb-nail figures should be compared horizontally by row, not vertically by column). Some of the children who reported that they could not see pictures or images in their imagination were nevertheless able to re-create similar representations over time. This strongly suggests that the processes of imagery in memory are non-representational. (As Edelman reasoned, memory's non-representational character results in later recollections varying in detail; the construction and re-construction from conceptual elements does not manifest in exact replication.) These results are shown graphically in Figs. 9 and $10 .^{8}$ Inspection shows that the frequency of similar images in the same interview (more recent memory) is in most cases greater than the frequency of similar images in longitudinal repeated interviews (older memory), findings that are in keeping with memory being inexact and nonrepresentational.

\section{Evidence in Support of the Hypothesis Posed by Research Question}

On the basis of what we detect in these interview analyses, the high coherence between imagery formed when talking, drawing and modelling supports the hypothesis that memory is dynamic, multi-modal and non-representational. The processes of memory create imagery which appear to the conscious mind as static and moving pictures. These afford reproduction into descriptions, drawings and models by processes of interpretation, but the memories, thoughts and images themselves are too dynamic to be representational in any meaningful sense. Rather than being a computer with stored information which can be recalled, the brain is a dynamic organ capable of creating concepts unconsciously from a myriad of elements (some everyday, some scientific) and able to share these with the conscious mind through imagery and other thought processes (such as inner speech), enabling the individual to visualise and reflect. These in turn may be shared with others through external speech, writing, drawing and

\footnotetext{
${ }^{8}$ In Figs. 6, 7, 8, 9 and 10, by 'more recent memory', we mean memory and imagery recalled or re-created during the same interview, i.e. within a period of several minutes to a few hours to a few days (the interviews being weather dependent and having to fit in with normal school activities), whereas by 'older memory', we mean memory and imagery recalled or re-created during the second of the longitudinal interviews a year later. The latter is influenced by learning and experience and, in many cases, inadvertently enhanced by the influence of repeated interviews and Socratic dialogue (see Bryce and Blown 2006; Blown and Bryce 2006).
} 
modelling by acts of representation. If memory was representational, one would expect all children to have recollections of pictures and similar images, but that is not the case. Several children had no knowledge of 'seeing' pictures in their imagination but were nevertheless able to explain, draw and model their concepts. This does suggest that there is some unconscious process by which conceptual interpretations cued by the researcher's questions are compared in the child's mind with memories of these concepts from a rich variety of sources. This could well involve unconscious imagery, or imagery unconscious to some children but not to others. But it does not have to involve representation in the form of a search for depictions as in scanning the pictures in an illustrated book or album. It may also be the case that we become more conscious of our imagination with development.

We did not set out to identify the format of children's mental representations as such in addressing our research question, mainly because we wished to keep an open mind about the possibility that imagery and memory are non-representational. We found no evidence of Pylyshyn's propositional representation since our methodology could not gauge the use of mental symbols in a propositional sense with sufficient refinement. There was, however, some evidence of non-representational memory as advocated by Edelman. Further research to ascertain which if any of the competing theories are correct awaits input from neuroscience. It is a question of considering alternative interpretations of evidence. We have put our argument in favour of memory and associated imagery being non-representational. While this is by no means conclusive, it does invite further enquiry into the nature of consciousness and whether or not representation is essential to thought including concept creation and re-creation. The data provided here is but a small fraction of the available evidence. As reported, there were 141 participants but space limited the selection to only a tenth of that number to be included as representative of the sample and included as protocols and drawings (see Figs. 2 and 3). However, as can be inferred from Table 1, a rich range of examples of both static and dynamic imagery was obtained across the age groups. Likewise, the drawings of similarity in ESM drawings over time (see Figs. 6, 7 and 8) are a small sample of the available data selected to illustrate the arguments in this paper.

\section{Discussion and Conclusion}

The results have shown how subjects recollected images of the shape and motion of the ESM during repeated interviews at intervals of minutes to several months apart. Although similar (in some cases almost identical), these representations have slight (often subtle) variations in drawings made within and between interviews ${ }^{9}$ on a given date. The evidence presented in

\footnotetext{
${ }^{9}$ For example, (1), in the case of the Earth, young children drawing the Earth as spherical, complete with 'Self' and 'Friend on the other side of the Earth' but with differences in the orientation of houses and the location of 'Self' and 'Friend'. Or, similar aged children drawing a dual-hemisphere Earth with 'Self' and 'Friend' standing on the surface of a truncated sphere; again with variations in the proportions of 'Ground', biosphere (the area where people live) and 'Sky' (see Fig. 6). Or, (2), in the case of the Sun, with young subjects drawing the Sun with prominences ('explosions'); and repeating the same structure a year later with one in the same place and another in a new location. We have also shown how children might draw the Sun with a flame-like structure around its periphery representing the corona; and repeat the same structure a year later; or with 18 alternately long and short rays drawn a year apart but with some at different angles to the Sun (see Fig. 7). Or (3), in the case of the Moon, children maintaining the shape but changing the orientation of its phases within and between longitudinal interviews, sometimes involving not only clockwise and anti-clockwise rotation in the plane of the page, but also top to bottom rotation perpendicular to the page (see Fig. 8).
} 
Figs. 2 and 3 support the first strand of the argument we set out in relation to the research question: that imagination has both conscious (representational) and non-conscious (nonrepresentational) forms as summarised in Figs. 4 and 5. Further evidence presented pictorially in Figs. 6, 7 and 8 and their related protocols support the second strand of the argument: that the basic patterns of concepts can be re-created hours, days, weeks or years between longitudinal interviews. These replications are approximations or analogues characterised by similarity rather than identical reproduction, as one would anticipate if memory was akin to a computer or imagery akin to a still or movie camera. The contrast between the frequency with which many children did not report images during any interview, yet proceeded to conceptualise effectively (explain/draw/model) (strand 3), also suggests that memory may be a non-representational process.

It is arguable that both facilities (unconscious imagery; and achieving similarity of representation over time) are related to pattern recognition - an evolutionary survival adaptation enabling early humans to detect predators and other dangerous creatures such as snakes and spiders. In modern times, this kind of skill enables astronomers to detect new features in the heavens such as comets and supernovae by comparing two images (mental or physical) over time. A comparable skill enables radiographers to detect changes in MRI images and X-rays to monitor injuries and disease by comparing images over time. Similar skills based on non-representative dynamic memory enable children to create detailed patterns of ESM shape and re-create them over time - changes in pattern resulting from learning and observation, as in the aforementioned examples.

Regarding the methodology used here, we consider that carefully conducted clinical interviews yield valid data which can give insights into how children think, including the processes of imagery, memory and metacognition. Neuroscience is also providing evidence in support of theories such as concept coexistence, but at this stage, the results are tentative. Neuroscientists see areas of the brain which are active during questioning, but cannot know what the neuronal interactivity is carrying, 'message-wise'. Some findings from neuroscience can be regarded as provisional: e.g. proof of coexistence and inhibition is inferred from delays in responses to questions, but as yet, there is no hard verification of these processes. Currently, clinical interviews, if conducted in a sensitive way by experienced researchers, remain the best method of investigating imagery, memory and metacognition (as researchers like Vosniadou and diSessa have productively demonstrated). The new theoretical account we have set out in this paper explains evidence that, rather than being compared with some form of mental model or template as a complete working entity, concepts appear to be dynamically constructed from selected elements created in memory: for example, images of the Earth having elements of physical shape including flatness or sphericity (see Fig. 1); images of the Sun having elements of shape and rays, and, with more senior pupils, possibly sunspots and flares; and across a wide age range, images of the Moon being dominated by elements related to the conflict between cultural and scientific interpretations of phases, maria and craters. An explanation has been offered which explains why children are able to describe, draw and model ESM in very similar ways months apart and how children are able to describe, draw and model ESM without conscious imagery.

Reflecting on the history of mental models in science education research, it is evident that many researchers have imagined mental models as existing in the minds of children; there to be revealed-by interview questions, by Socratic dialogue or by (future) neuroscience? However, perhaps their existential status has been exaggerated. Are they 
only devices which serve to categorise children's concepts-researchers' constructions imposed (however carefully) upon a wide range of the elements of a child's knowledge in a particular domain? It may be that some researchers contemplate both possibilities. Also, it is to be hoped that developments in brain research will soon clarify the relationships between the conscious and unconscious processes which underlie people's responses to questioning. It would not be appropriate to pursue this line of argument further at this stage. Fresh investigations are needed to seek wider support for the ideas contained here. As a first step, we consider that the considerations raised in this paper should be explored in relation to other scientific topics and concepts which figure in children's learning.

\section{Compliance with ethical standards}

Conflict of Interest The authors declare that they have no conflict of interest.

Open Access This article is distributed under the terms of the Creative Commons Attribution 4.0 International License (http://creativecommons.org/licenses/by/4.0/), which permits unrestricted use, distribution, and reproduction in any medium, provided you give appropriate credit to the original author(s) and the source, provide a link to the Creative Commons license, and indicate if changes were made.

\section{References}

Aikenhead, G. S. (1996). Science education: border crossing into the subculture of science. Studies in Science Education, 27, 1-52.

Aikenhead, G. S. (1997). Toward a first nations cross-cultural science and technology curriculum. Science Education, 81, 217-238.

Anning, A., \& Ring, K. (2005). Making sense of children's drawings. Milton Keyes: The Open University Press.

Artberry, M. E., Craver-Lemley, C., \& Reeves, A. (2002). Visual imagery is not always like visual perception. Commentary to Pylyshyn, 2002, 183-184.

Ausubel, D. P. (2000). The acquisition and retention of knowledge: a cognitive view. Boston: Kluwer.

Blown, E. J., \& Bryce, T. G. K. (2006). Knowledge restructuring in the development of children's cosmologies. International Journal of Science Education, 28(12), 1411-1462.

Blown, E. J., \& Bryce, T. G. K. (2010). Conceptual coherence revealed in multi-modal representations of astronomy knowledge. International Journal of Science Education, 32(1), 31-67.

Blown, E. J., \& Bryce, T. G. K. (2013). Thought-experiments about gravity in the history of science and in research into children's thinking. Science \& Education, 22(3), 419-483.

Blown, E. J., \& Bryce, T. G. K. (2017). Switching between everyday and scientific language. Research in Science Education, 47, 621-653.

Blown, E. J., \& Bryce, T. G. K. (2018). The enduring effects of early-learned ideas and local folklore on children's astronomy knowledge. Research in Science Education. Online First. https://doi.org/10.1007 /s11165-018-9756-1.

Barsalou, L. W. (1999). Perceptual symbol systems. Behavioral and Brain Sciences, 22, 577-660.

Barsalou, L. W. (2003). Situated simulation in the human perceptual system. Language \& Cognitive Processes, $18(5 / 6), 513-562$.

Barsalou, L. W. (2008). Grounded cognition. Annual Review of Psychology, 59, 617-645.

Bartolomeo, P., \& Chokron, S. (2002). Can we change our vantage point to explore imaginal neglect? Commentary to Pylyshyn, 2002, 184-185.

Bishop, G. (1996). Maui and the Sun. Auckland: North-South Books.

Brault Foisy, L.-M., Potvin, P., Riopel, M., \& Masson, S. (2015). Is inhibition involved in overcoming a common physics misconception in mechanics? Trends in Neuroscience and Education, 4(1-2), 26-36. https://doi. org/10.1016/j.tine.2015.03.001. 
Brewer, W. F., Herdrich, D. J., \& Vosniadou, S. (1987, January). A cross-cultural study of children's development of cosmological models: Samoan and American data. Paper presented at the Third International Conference on Thinking. HI: Honolulu.

Bryce, T. G. K., \& Blown, E. J. (2006). Cultural mediation of children's cosmologies: a longitudinal study of the astronomy concepts of Chinese and New Zealand children. International Journal of Science Education, 28(10), 1113-1160.

Bryce, T. G. K., \& Blown, E. J. (2007). Gender effects in children's development and education. International Journal of Science Education, 29(13), 1655-1678.

Bryce, T. G. K., \& Blown, E. J. (2012). The novice-expert continuum in astronomy knowledge. International Journal of Science Education, 34(4), 545-587.

Bryce, T. G. K., \& Blown, E. J. (2013). Children's concepts of the shape and size of the Earth, Sun and Moon. International Journal of Science Education, 35(3), 388-446.

Bryce, T. G. K., \& Blown, E. J. (2016). Manipulating models and grasping the ideas they represent. Science \& Education, 25(1), 47-93.

Burgess, N. (2002). Spatial models of imagery for remembered scenes are more likely to advance (neuro)science than symbolic ones. Commentary to Pylyshyn, 2002, 185-186.

Cakir, M. (2008). Constructivist approaches to learning in science and their implications for science pedagogy: a literature review. International Journal of Environmental \& Science Education, 3(4), 193-206.

Carey, S. (1991). Knowledge acquisition: enrichment or conceptual change? In S. Carey \& R. Gelman (Eds.), The epigenesis of mind: essays on biology and cognition (pp. 257-291). Hillsdale, NJ: Erlbaum.

Carey, S. (2009). The origin of concepts. Oxford: Oxford University Press.

Carroll, L. (2003). Alice in wonderland. Bath: Paragon (Original work published 1865).

Chaterjee, A. (2002). Pictures, propositions, and primitives in the head. Commentary to Pylyshyn, 2002, 186187.

Chi, M. T. H., \& Slotta, J. D. (1993). The ontological coherence of intuitive physics. Cognition and Instruction, $10(2 \& 3), 249-260$.

Davids, K., \& Bennett, S. (1998). The dynamical hypothesis: The role of biological constraints on cognition. Behavioral and Brain Sciences, 21(2), 636.

diSessa, A. A. (1983). Phenomenology and the evolution of intuition in D. Gentner \& A. L. Stevens (Eds.). Mental models. Hillsdale, NJ: Lawrence Earlbaum pp. 15-34.

diSessa, A. A. (1988). Knowledge in pieces. In G. Forman \& P. B. Pufall (Eds.), Constructivism in the computer age (pp. 49-70). Hillsdale, NJ: Lawrence Erlbaum.

diSessa, A. (1993). Toward an epistemology of physics. Cognition and Instruction, 10, 105-225. https://doi. org/10.1080/07370008.1985.9649008.

diSessa, A. A. (2008). A bird's eye view of the "pieces" vs. "coherence" controversy from the "pieces" side of the fence. In S. Vosniadou (Ed.), International handbook of research on conceptual change (pp. 35-60). New York: Routledge.

diSessa, A. A. (2017). Conceptual change in a microcosm: comparative analysis of a learning event. Human Development, 60, 1-37. https://doi.org/10.1159/000469693.

Donaldson, M. (1978). Children's minds. London: Fontana.

Dresp-Langley, B. (2012). Why the brain knows more than we do: non-conscious representations and their role in the construction of conscious experience. Brain Sciences, 2, 1-21.

Driver, R. H. (1981). Pupils' alternative frameworks in science. European Journal of Science Education, 3(1), 93-101.

Driver, R., Asoko, H., Leach, J., Mortimer, E., \& Scott, P. (1994). Constructing scientific knowledge in the classroom. Educational Researcher, 23(7), 5-12 http://www.jstor.org/stable/1176933.

Driver, R., \& Easley, J. (1978). Pupils and paradigms: a review of literature related to concept development in adolescent science students. Studies in Science Education, 5, 61-84.

Driver, R., Guesne, E., \& Tiberghien, A. (Eds.). (1985). Children's ideas in science. Milton Keynes, UK: Open University Press.

Duffy, B. (2006). Supporting creativity and imagination in the early years. Maidenhead, Berkshire: Open University Press.

Duit, R. (1994, September). Conceptual change approaches in science education. Paper presented at the symposium on conceptual change: Freidrich Schiller University of Jena.

Dunbar, K. N., Fugelsang, J. A., \& Stein, C. (2007). Do naïve theories ever go away? Using brain and behaviour to understand changes in concepts. In M. C. Lovett \& P. Shah (Eds.), Proceedings of the $33^{\text {rd }}$ Carnegie symposium on cognition, thinking with data (pp. 193-206). NJ, Erlbaum: Mahwah.

Edelman, G. (1989). The remembered present: a biological theory of consciousness. New York: Basic Books.

Edelman, G. (2001). Consciousness: the remembered present. Annals of New York Academy of Sciences, 929, 111-122. 
Edelman, G. M. (2005). Wider than the sky: a revolutionary view of consciousness. London: Penguin.

Edelman, G. M. (2006). Second nature: brain science and human knowledge. New Haven: Yale University Press.

Edelman, G. M., \& Changeux, J.-P. (2001). The brain. New Brunswick, NJ: Transaction Publishers.

Freeman, W. J., \& Skarda, C. A. (1990a). Chaotic dynamics versus representationalism. Behavioral and Brain Sciences, 13, 167-168.

Freeman, W. J., \& Skarda, C. A. (1990b). Representations: Who needs them? In J. L. McGaugh, N. M. Weinberger, \& G. Lynch (Eds.), Brain organization and memory cells, systems and circuits. Oxford: Guildford.

Fugelsang, J. A., \& Dunbar, K. N. (2005). Brain-based mechanisms underlying complex causal thinking. Neuropsychologia, 43, 1204-1213.

Funk, M., Brugger, P., \& Wilkening, F. (2005). Motor processes in children's imagery: the case of mental rotation of hands. Developmental Science, 8, 402-408.

Gardner, H. G. (1991). The unschooled mind. New York: Basic.

Gascoine, L., Higgins, S., \& Wall, K. (2016). The assessment of metacognition in children aged 4-16 years: a systematic review. The Review of Education, 5(1), 3-57.

Gentner, D., \& Stevens, A. (1983). Mental models. Hillsdale, NJ: Lawrence Erlbaum.

Goldenberg, G. (2002). Loss of visual imagery: neuropsychological evidence in search for a theory. Commentary to Pylyshyn, 2002, 191.

Goldin-Meadow, S. (2017). What the hands can tell us about language emergence. Psychonomic Bulletin \& Review, 24(1), 213-218. https://doi.org/10.3758/s13423-016-1074-x,Abstract,PDF.

Goodnow, J. (1977). Children's drawing. London: Fontana.

Hoenig, K., Sim, E. J., Bochev, V., Herrnberger, B., \& Kiefer, M. (2008). Conceptual flexibility in the human brain: dynamic recruitment of semantic maps from visual, motor and motion-related areas. Journal of Cognitive Neuroscience, 20(10), 1799-1814. https://doi.org/10.1162/jocn.2008.20123.

Horgan, J. (2014). My testy encounter with the Late, Great Gerald Edelman. Scientific American, May 22.

Johnson-Laird, P. N. (1983). Mental models. Cambridge, MA: Harvard University Press. Psychology, 25(2), $131-138$.

Kahana, M. J. (2012). Foundations of human memory. Oxford: Oxford University Press.

Kiefer, M., \& Pulvermüller, F. (2011). Conceptual representations in mind and brain: theoretical developments, current evidence and future directions. Cortex, 04/2011.

Leonard, M. J., Kalinowski, S. T., \& Andrews, T. C. (2014). Misconceptions yesterday, today, and tomorrow. CBE Life Sciences Education, 13(2), 179-186.

Malchiodi, C. A. (1998). Understanding children's drawings. London: Guilford.

Mareschal, D. (2016). The neuroscience of conceptual learning in science and mathematics. Current Opinion in Behavioral Sciences, 10, 114-118.

Masson, S., Potvin, P., Riopel, M., \& Brault Foisy, L.-M. (2014). Differences in brain activation between novices and experts in science during a task involving a common misconception in electricity. Mind, Brain, and Education, 8(1), 44-55. https://doi.org/10.1111/mbe.12043.

National Research Council (2007). Taking science to school: Learning and teaching science in grades K-8. Washington, DC: National Academies Press. https://doi.org/10.17226/11625.

Nussbaum, J. (1979). Children's conceptions of the earth as a cosmic body: a cross age study. Science Education, $63,83-93$.

Nussbaum, J., \& Novak, J. D. (1976). An assessment of children's concepts of the Earth utilizing structured interviews. Science Education, 60, 535-550.

Paivio, A., Clark, J. M., Digdon, N., \& Bons, T. (1989). Referential processing: reciprocity and correlates of naming and imaging. Memory \& Cognition, 17(2), 163-174.

Paivio, A. (1971). Imagery and verbal processes. New York: Holt, Rinehart \&Winston.

Paivio, A. (1986). Mental representations: a dual coding approach. New York: Oxford Univ. Press.

Petitto, L.-A. \& Dunbar, K. N. (2009). Educational neuroscience: new discoveries from bilingual brains, scientific brains, and the educated mind. Mind, Brain, and Education. 2009 December 1; 3(4): 185-197. https://www.ncbi.nlm.nih.gov/pmc/articles/PMC3338206/

Piaget, J. (1929). The child's conception of the world. London, UK: Routledge \& Kegan Paul.

Piaget, J. (1930). The child's conception of physical causality. London, UK: Routledge \& Kegan Paul.

Potvin, P. (2017). The coexistence claim and its possible implications for success in teaching for conceptual "change". European Journal of Science and Mathematics Education, 5(1), 55-66.

Pylyshyn, Z. W. (2002). Mental imagery: in search of a theory. Behavioral and Brain Sciences, 25, 157-238.

Rahman, N. A. (2004). Physics teachers' strategies and reflective ability for addressing pupils' misconceptions in the classroom. Paper presented at the British Educational Research Association Conference, Manchester, 2014. 
Rose, S. (2006). The 21st century brain: explaining, mending and manipulating. London: Jonathan Cape.

Rose, S. (2007). Thinking allowed: review of second nature: brain science and human knowledge by Gerald M. Edelman. The Guardian, Saturday 17 February.

Schacter, D. L. (1996). Searching for memory: the brain, the mind, and the past. New York: Basic Books.

Seth, A. K. (2014). Darwin's neuroscientists: Gerald M. Edelman, 1929-2014. Frontiers in Psychology, 14 August. doi: https://doi.org/10.3389/fpsyg.2014.00896

Shulman, A., \& Valcarcel, J. (2012). Scientific knowledge suppresses but does not supplant earlier intuitions. Cognition, 124, 209-215.

Siegal, M., Butterworth, G., \& Newcombe, P. A. (2004). Culture and children's cosmology. Developmental Science, 7(3), 308-324. https://doi.org/10.1111/j.1467-7687.2004.00350.x.

Smith, J., diSessa, A., \& Rochelle, J. (1993). Misconceptions reconceived: a constructivist analysis of knowledge in transition. Journal of the Learning Sciences, 3, 115-163.

Solomon, J. (1983). Messy, contradictory and obstinately persistent: a study of children's out-of-school ideas about energy. School Science Review, 65, 225-229.

Stanford. (2015). Stanford encyclopedia of philosophy. http://plato.stanford.edu/

Taber, K. S. (2000). Multiple frameworks? Evidence of manifold conceptions in individual cognitive structure. International Journal of Science Education, 22(4), 399-417.

Thomas, N. J. T. (2002). The false dichotomy of imagery. Commentary to Pylyshyn, 2002, 211.

Thomas, N. J. T. (2014). Dual coding and common coding theories of memory. Supplement to mental imagery in Stanford encyclopedia of philosophy. See at http://plato.stanford.edu/entries/mental-imagery/theoriesmemory.html

Tulving, E. (1972). Episodic and semantic memory. In E. Tulving \& W. Donaldson (Eds.), Organisation of memory (pp. 381-403). Academic: New York.

Tulving, E. (2002). Episodic memory from mind to brain. Annual Review of Psychology, 53, 1-25.

van Gelder, T. (1998). The dynamical hypothesis in cognitive science. The Behavioral and Brain Sciences, 21(5), 615-628.

Vosniadou, S. (2014). Examining cognitive development from a conceptual change point of view: the framework theory approach. European Journal of Developmental Psychology, 11(6), 645-661. https://doi.org/10.1080 $/ 17405629.2014 .921153$.

Vosniadou, S., \& Brewer, W. F. (1992). Mental models of the Earth: a study of conceptual change in childhood. Cognitive Psychology, 24(4), 535-585.

Vosniadou, S., \& Brewer, W. F. (1994). Mental models of the day/night cycle. Cognitive Science, 18(1), 123183.

Vosniadou, S., Ioannides, C., Dimitrakopoulou, A., \& Papademetriou, E. (2001). Designing learning environments to promote conceptual change in science. Learning and Instruction, 11(4-5), 381-419.

Vosniadou, S., \& Skopeliti, I. (2014). Conceptual change from the framework theory side of the fence. Science \& Education, 23, 1427-1445.

Vosniadou, S., Vamvakoussi, X., \& Skopeliti, I. (2008). The framework theory approach to the problem of conceptual change. In S. Vosniadou (Ed.), International handbook of research on conceptual change (pp. 35-60). New York: Routledge.

Vygotsky, L. S. (1986). Thought and language (A. Kozulin, Ed. \& Trans.). Cambridge, MA: MIT Press. (Original work published 1934).

Wheeler, M. A., Stuss, D. T., \& Tulving, E. (1997). Toward a theory of episodic memory. Psychological Bulletin, $121,331-354$.

Zhang, Y. (2011). 365 Nights stories. Shanghai: People's Fine Arts Publishing House.

Publisher's Note Springer Nature remains neutral with regard to jurisdictional claims in published maps and institutional affiliations. 